\title{
Free energy of a Lovelock holographic superconductor
}

\author{
Ligeia Aranguiz ${ }^{1,2, a}$, Olivera Miskovic ${ }^{2, b}$ \\ ${ }^{1}$ Universidad Técnica Federico Santa María, Casilla 110-V, Valparaíso, Chile \\ ${ }^{2}$ Instituto de Física, Pontificia Universidad Católica de Valparaíso, Casilla 4059, Valparaíso, Chile
}

Received: 23 January 2014 / Accepted: 10 July 2014 / Published online: 7 August 2014

(C) The Author(s) 2014. This article is published with open access at Springerlink.com

\begin{abstract}
We study thermodynamics of black hole solutions in Lanczos-Lovelock anti-de Sitter gravity in $d+1$ dimensions coupled to nonlinear electrodynamics and a Stückelberg scalar field. This class of theories is used in the context of gauge/gravity duality to describe a hightemperature superconductor in $d$ dimensions. A larger number of coupling constants in the gravitational side is necessary to widen the domain of validity of physical quantities in dual quantum field theory (QFT). We regularize the gravitational action and find the finite conserved quantities for a planar black hole with scalar hair. Then we derive the quantum statistical relation in the Euclidean sector of the theory, and we obtain the exact formula for the free energy of the superconductor in the holographic QFT. Our result is analytic and it includes the effects of backreaction of the gravitational field. We further discuss on how this formula could be used to analyze second order phase transitions through the discontinuities of the free energy, in order to classify holographic superconductors in terms of the parameters in the theory.
\end{abstract}

\section{Introduction}

The proposal of Maldacena about an equivalence between the anti-de Sitter (AdS) gravity and the conformal field theory (CFT) in a smaller spacetime dimension [1-4] has brought to many successful applications of this correspondence to strongly coupled quantum systems. This conjectured holographic-type duality between two theories is still the only approach to field theories where strong coupling results are calculated exactly from its gravitational weakly coupled dual system.

We are interested in studying thermal phase transitions in quantum field theories in the context of AdS/CFT correspondence. The temperature in holographic quantum field

\footnotetext{
a e-mail: ligeia.aranguiz@postgrado.usm.cl

be-mail: olivera.miskovic@ucv.cl
}

theory (QFT) is identified with the Hawking temperature of the black hole in AdS gravity which arises from quantum effects near the event horizon. A phase transition occurs in a theory when its effective potential changes as a consequence of temperature variations and leads to vacuum instabilities. We assume that the temperatures are high enough in order to favor transitions due to temperature fluctuations, and not the ones that happen because of quantum fluctuations. In particular, we shall focus on high-temperature superconductors whose critical temperature, $T_{\mathrm{c}}$, is above the limit of applicability of the Bardeen-Cooper-Schrieffer (BCS) theory, and we use the gauge/gravity duality to describe phase transitions as a fundamental phenomenon and not an effective one, as in the BCS model.

Typical features of the superconducting phase in a superconductor are related to its response to electric and magnetic fields, such as the infinite conductivity (i.e., superconductivity) and the expulsion of magnetic field from it (the Meissner effect). Thus, in order to obtain a holographic superconductor in $d$ dimensions, the dual $\operatorname{AdS}_{d+1}$ space has to contain an electrically charged black hole coupled to charged matter, for example, a complex scalar field [5]. Then the electromagnetic field becomes a source for an electromagnetic current in the boundary QFT and the scalar field couples to an order parameter. In addition, on the gravity side a charged black hole will develop a scalar hair as the Hawking temperature increases, which will correspond to a phase transition of a superconductor on the QFT side. The holographic procedure enables to compute dynamical transport properties of a such superconductor directly from the gravitational dynamics.

Second order phase transitions are present in holographic QFT dual to general relativity minimally coupled to the Maxwell field and one complex scalar. Even in this simplest case, in order to obtain information about a holographic superconductor, one faces the technical problem of solving nonlinear field equations of matter interacting with gravity in AdS space. The easiest way to circumvent this problem is to take a probe limit, that is, to study the dynamics of the 
matter fields in the black hole background, i.e., neglecting the gravitational backreaction. Then one might use numerical methods to solve the equations of motion. For example, in [6], the authors study electric and magnetic fields in a holographic Stückelberg superconductor [7] with a minimally coupled scalar field in a four-dimensional planar AdS black hole background. They calculate the critical exponent, superconductivity, the energy gap, free energy and specific heat in this theory using numerical methods. A similar problem in the background of a planar black hole with a monopole and a multiplet of complex scalar fields in four dimensions was discussed in [8]. In $d$ dimensions, a more general system has been analyzed in $[9,10]$, where a number of physical quantities was obtained for the Stückelberg holographic superconductor with the scalar potential $\Psi^{n}$, finding first order $(n>2)$ and second order $(n=2)$ phase transitions. A linear combination of the potentials with different $n$ was also discussed. In the same context, but adding Weyl corrections, it was found in [11] that the critical exponent does not depend on the Weyl parameter, which just confirms its universal value $1 / 2$ that does not depend on the details of the dynamics of the system. It is worthwhile mentioning that the holographic models of high-temperature superconductivity also successfully explain the large ratio of the energy gap to critical temperature compared to conventional superconductors $[5,6]$.

A semi-analytic approach to phase transitions in four dimensions is beautifully described by Gubser $[12,13]$ as a symmetry breaking effect, where a superconductor is formed near the black hole horizon. Different types of superconductors (s-wave [14,15] and p-wave [16,17], whose order parameters are the scalar and the vector, respectively) are explained using Abelian and non-Abelian gauge fields. Analytic calculations in the asymptotic region near the phase transition point are performed in five-dimensional AdS gravity in [18] for the Stückelberg equations of motion that are solved in the probe limit. The backreaction of the hairy charged black hole in four dimensions has been included in [19], where a semi-analytic solution is obtained by matching smoothly the near-horizon series with the asymptotic solution at the matching point located between the horizon and the infinity.

In order to explain different types of holographic high- $T_{\mathrm{c}}$ superconductors discovered in nature, which do not fit any theoretical description, one needs a broad class of different CFTs coming from AdS gravities in the bulk. A massive scalar field in AdS space can give as many unitary CFTs on the boundary as there are possible boundary conditions, but in general there are just few ones (for example Dirichlet, Neumann and mixed boundary conditions) [20]. This is not enough to explain the variety of properties of these superconductors. Thus, in higher dimensions, it is natural to consider a generalization of general relativity in AdS space, that is, higher-curvature Lanczos-Lovelock AdS gravities [21-23] that depend on a family of coupling constants and still possess second order field equations in the metric field. The presence of these couplings can change physical properties of holographic quantities, such as critical temperature and transport coefficients ratios. In recent work on holographic superconductors with electromagnetic and charged scalar fields that deal with nonlinear gravitational effects, EinsteinGauss-Bonnet (EGB) AdS gravity quadratic in curvature, was studied in an arbitrary dimension $[24,25]$ in the probe limit and using the numerical methods. A typical result is that the Gauss-Bonnet (GB) coupling decreases the critical temperature of the superconductor and thus makes the condensation harder [26-31]. In these references, an analytical approach to the condensation in a holographic dual to EGB gravity that includes an effect of the backreaction of black holes in five dimensions was also discussed. Again, inclusion of the backreaction of the gravitational field makes the condensation harder in these theories. Interestingly, in [32] it was found that for very strong GB couplings, the critical temperature begins to increase. Another technique to analytically study the critical phenomena, based on the variational method for the Sturm-Liouville operator, was used in [33]. Different aspects of a holographic superconductor with the GB term were also analyzed in $[19,24,25,29-32,34-40]$, showing that the higher-order curvature corrections can modify the universal ratio of the gap in the frequency-dependent conductivity to critical temperature $\left(\omega_{\mathrm{g}} / T_{\mathrm{c}}=8\right)$. Similarly, in dual holographic hydrodynamics, the universal ratio bound of the shear viscosity to entropy density, $\eta / s \leq 1 / 4 \pi$ [41], can be changed by higher-order curvature terms (see, e.g. [42]). Another way to modify the ratio $\omega_{\mathrm{g}} / T_{\mathrm{c}}$ is in dilaton extensions of holographic superconductors [43].

On the other hand, gauge theories which are described by nonlinear actions for Abelian or non-Abelian connections have also become standard in the context of superstring theory and provide richer physics in holographic theories. For example, introduction of the Born-Infeld electrodynamics (in presence of EGB AdS black holes) affects the formation of the scalar hair since it changes the transition point of the phase transition from the second order to the first order $[24,25]$. Also, a power-Maxwell electromagnetic field in the background of the Schwarzschild AdS black hole makes the scalar hair harder to condensate for larger power parameter [44]. In both cases the critical exponent of the system remains the same as in the Landau-Ginzburg mean field theory, that is, $1 / 2$, and the same happens for a large class of nonlinear electrodynamics models. Inclusion of a dynamical electromagnetic field in holographic superconductors was discussed in [45], which was crucial to obtain properties such as the Meissner effect. Notice that, although the magnetic field makes the condensate harder to form, a negative GB coupling enhances the condensation when the field is not too strong [46]. 
Newer results on nonlinear effects in holographic superconductors can be found in [47-50]. For more on holographic superconductors, see the comprehensive lectures in, e.g., $[51,52]$.

In our approach, we are interested in analytic study of the effects of higher-order gravitational terms in AdS gravity and nonlinear electrodynamics on phase transitions in high- $T_{\mathrm{c}}$ holographic superconductors of the Stückelberg type, which includes the backreaction of the black holes. The goal is to find an exact formula for the free energy of a superconductor in an arbitrary dimension $d$ that is UV finite and it depends on at most on $[d / 2]$ gravitational coupling parameters of the Lanczos-Lovelock action and two arbitrary functions stemming from the NED and Stückelberg actions. In the course of this, we shall renormalize the IR sector of $\mathrm{AdS}_{d+1}$ gravity, which corresponds to a UV renormalization of a holographic quantum effective action. As a result, we shall obtain the result that the free energy of the system at finite $T$ satisfies the quantum statistical relation. Since the free energy and the corresponding statistical partition function contain all thermodynamic information about the holographic quantum system, this formula will open the possibility to analyze the local and global minima of the thermodynamic potential and, in this way, detect all possible phase transitions in the theory depending on the values of coupling constants, similarly as in the Landau-Ginzburg description of superconductivity.

In this paper, we shall focus on the first part of the above problem and only discuss the second part, which is work in progress.

\section{Lanczos-Lovelock AdS gravity and the equations of motion}

The Lanczos-Lovelock (LL) gravity [21-23] in $D=d+1$ dimensions is described by an action polynomial in the Riemann curvature in such a way that its equations of motion still keep properties of general relativity and give rise to at most second order field equations in the metric. This happens because the $p$ th term of the LL polynomial, $L_{p}$, is a dimensional continuation of the Euler density in $2 p$ dimensions,

$$
\begin{aligned}
& I_{\mathrm{LL}}=\frac{1}{2 \kappa^{2}} \int d^{d+1} x \sqrt{-g} \sum_{p=0}^{[d / 2]} \alpha_{p} L_{p}, \\
& L_{p}=\frac{1}{2^{p}} \delta_{\nu_{1} \ldots \nu_{2 p}}^{\mu_{1} \ldots \mu_{2 p}} R_{\mu_{1} \mu_{2}}^{\nu_{1} \nu_{2}} \ldots R_{\mu_{2 p-1} \mu_{2 p}}^{\nu_{2 p-1} \nu_{2 p}},
\end{aligned}
$$

where $\delta_{v_{1} \ldots \nu_{2 p}}^{\mu_{1} \ldots \mu_{2 p}}=\delta_{v_{1}}^{\mu_{1}} \delta_{v_{2}}^{\mu_{2}} \ldots \delta_{\nu 2 p}^{\mu_{2 p}}+\cdots$ denotes the completely antisymmetric product of $2 p$ Kronecker's deltas. In our notation, the metric field $g_{\mu \nu}$ is mostly positive and the Riemann curvature reads $R_{\nu \alpha \beta}^{\mu}=\partial_{\alpha} \Gamma_{v \beta}^{\mu}-\partial_{\beta} \Gamma_{\nu \alpha}^{\mu}+$ $\Gamma_{\lambda \alpha}^{\mu} \Gamma_{v \beta}^{\lambda}-\Gamma_{\lambda \beta}^{\mu} \Gamma_{v \alpha}^{\lambda}$.
The last non-vanishing term in the sum, $L_{d+1}$, is the Euler topological invariant that does not contribute to the dynamics, so it has not been included in the series. The terms with $L_{2 p>d+1}$ are identically vanishing. The strength of gravitational interaction is determined by the Newton constant $G_{N}=\kappa^{2} / 8 \pi$. The gravitational part of the theory depends on the set of the coupling constants, $\alpha_{p}$, of dimension [length] ${ }^{2 p-2}$. The first term in the LL polynomial is constant, $L_{0}=1$, so that $\alpha_{0}=-2 \Lambda$ is related to the cosmological constant, that we shall assume to be negative, $\Lambda=-d(d-1) / 2 \ell^{2}$. Here, $\ell$ is the AdS radius. The linear term in the curvature is the Einstein-Hilbert term $L_{1}=R$, normalized as $\alpha_{1}=1$. Other terms can be seen as higher-order curvature corrections to general relativity. The simplest, quadratic correction is given by the GB term $L_{2}=R^{2}-4 R_{\mu \nu} R^{\mu \nu}+R_{\mu \nu \lambda \sigma} R^{\mu \nu \lambda \sigma}$ with the coupling $\alpha_{2}=\alpha$. Even though we assume that the constants $\alpha_{p}$ are arbitrary for $p \geq 2$, from the point of view of the AdS/CFT correspondence, there are restrictions on their values related to preserving of the causality in the boundary of asymptotically AdS spacetime [53-55].

The metric is coupled to the Abelian gauge field $A_{\mu}(x)$ with the associated field strength $F_{\mu \nu}=\partial_{\mu} A_{\nu}-\partial_{\nu} A_{\mu}$ through the quadratic invariant $F^{2}=g^{\mu \alpha} g^{\nu \beta} F_{\mu \nu} F_{\alpha \beta}$. In order to include nonlinear effects, we choose the electromagnetic field described by nonlinear electrodynamics (NED) whose Lagrangian density is an arbitrary function in the invariant $F^{2}$,

$I_{\mathrm{NED}}=\frac{1}{2 \kappa^{2}} \int d^{d+1} x \sqrt{-g} \mathcal{L}\left(F^{2}\right)$.

The gravitational and NED fields are coupled to a complex scalar field $\hat{\Psi}=\Psi \mathrm{e}^{i p}$, where nonlinear effects are introduced through a non-minimal coupling of the Stückelberg action [7],

$$
\begin{aligned}
I_{\mathrm{S}}= & \frac{1}{2 \kappa^{2}} \int d^{d+1} x \sqrt{-g} \\
& \times\left[-\frac{1}{2}(\partial \Psi)^{2}-\frac{1}{2} m^{2} \Psi^{2}-\frac{1}{2} \mathcal{F}(\Psi)(\partial p-A)^{2}\right]
\end{aligned}
$$

Here, $\Psi(x)$ and $p(x)$ are real scalar fields, and $\mathcal{F}(\Psi)$ is an arbitrary real function that satisfies $\mathcal{F}(0)=0$ and $\mathcal{F}(\Psi) \geq 0$ in order to ensure positivity of the kinetic term for $p$. The minimal coupling between the scalar and EM fields is recovered by choosing the interaction as $\mathcal{F}_{\text {minimal }}(\Psi)=\Psi^{2}$.

The total bulk action,

$I_{0}=I_{\mathrm{LL}}[g]+I_{\mathrm{NED}}[g, A]+I_{\mathrm{S}}[g, A, \Psi, p]$,

depends on the set of constants in the gravity part, $\kappa, \Lambda$ and $\alpha_{p}$, and two arbitrary functions $\mathcal{L}\left(F^{2}\right)$ and $\mathcal{F}(\Psi)$ determining completely the matter couplings. 
Our goal is to understand for which gravitational parameter range and what interaction with the matter (within a chosen class of theories) it is possible to have a phase transition of second order in holographically dual QFT.

Provided the boundary terms have been added to the bulk action, which we shall discuss later in detail, the action reaches an extremum for the following equations of motion:

$$
\begin{aligned}
& \delta g^{\mu \nu}:-\sum_{p=0}^{[d / 2]} \frac{\alpha_{p}}{2^{p+1}} g_{\nu \lambda} \delta_{\mu \mu_{1} \ldots \mu_{2 p}}^{\lambda \nu_{1} \ldots \nu_{2 p}} R_{\nu_{1} \nu_{2}}^{\mu_{1} \mu_{2}} \ldots R_{\nu_{2 p-1} \nu_{2 p}}^{\mu_{2 p-1} \mu_{2 p}}=T_{\mu \nu}, \\
& \delta A_{\mu}: \nabla_{\nu}\left(4 F^{\mu \nu} \frac{d \mathcal{L}}{d F^{2}}\right)=-\mathcal{F}(\Psi)\left(\nabla^{\mu} p-A^{\mu}\right), \\
& \delta \Psi:\left(\nabla^{2}-m^{2}\right) \Psi=\frac{1}{2} \frac{d \mathcal{F}}{d \Psi}(\nabla p-A)^{2}, \\
& \delta p: \nabla_{\mu}\left[\mathcal{F}(\Psi)\left(\nabla^{\mu} p-A^{\mu}\right)\right]=0,
\end{aligned}
$$

where $\nabla_{\mu}$ is covariant derivative with respect to the affine connection $\Gamma_{\nu \lambda}^{\mu}$. The symmetric energy-momentum tensor for the matter fields, conveniently normalized as $T_{\mu \nu}=$ $-\frac{4 \kappa^{2}}{\sqrt{-g}} \frac{\delta\left(I_{\mathrm{NED}}+I_{\mathrm{S}}\right)}{\delta g^{\mu \nu}}$, has the form

$$
\begin{aligned}
T_{\mu \nu}= & \frac{1}{2} g_{\mu \nu} \mathcal{L}+\frac{d \mathcal{L}}{d F^{2}} 2 F_{\mu \lambda} F_{\nu}^{\lambda} \\
& -\frac{1}{4} g_{\mu \nu}\left[(\partial \Psi)^{2}+m^{2} \Psi^{2}+\mathcal{F}(\Psi)(\partial p-A)^{2}\right] \\
& +\frac{1}{2} \partial_{\mu} \Psi \partial_{\nu} \Psi+\frac{1}{2} \mathcal{F}(\Psi)\left(\partial_{\mu} p-A_{\mu}\right)\left(\partial_{\nu} p-A_{\nu}\right) .
\end{aligned}
$$

In order to ensure a non-negative energy density of the matter, we impose the weak energy condition on the energymomentum tensor, $w=-T_{\mu \nu} u^{\mu} u^{\nu} \geq 0$, for a time-like unit vector $u^{\mu}$.

The system of equations (5) can be simplified by noticing that the last equation is not independent from the others and can be consistently eliminated by fixing the $U(1)$ gauge symmetry, $p(x) \rightarrow p(x)+\alpha(x)$. From now on, we set $p(x)=0$.

In order to have an asymptotically AdS spacetime, we assume that there exists the $\operatorname{AdS}$ vacuum $\left(T_{\mu \nu}=0\right)$ with constant curvature globally, $R_{\mu_{1} \mu_{2}}^{\nu_{1} \nu_{2}}=-\frac{1}{\ell_{\text {eff }}^{2}} \delta_{\mu_{1} \mu_{2}}^{\nu_{1} \nu_{2}}$, with an effective AdS radius, $\ell_{\text {eff }}$. Plugging in this condition in the gravitational equation (5) gives rise to a polynomial

$0=\sum_{p=0}^{[d / 2]} \frac{\alpha_{p}}{(d-2 p) !}\left(-\ell_{\mathrm{eff}}^{-2}\right)^{p}$

which has at most $[d / 2]$ different roots $1 / \ell_{\mathrm{eff}}^{2}$ for a given set of the coefficients $\left\{\alpha_{p}\right\}$.

Now we want to describe a charged AdS black hole solution to (5). It is well known that black holes exist in pure LL AdS gravity [56-59] and in LL AdS gravity coupled to NED, in particular in Born-Infeld electrodynamics [60]. Their thermodynamics has also been studied [61,62]. For a recent review on Lovelock gravities, see, e.g., [63], and in the context of holography [64].

\section{Charged planar black hole in Einstein-Gauss-Bonnet gravity}

We start from the EGB AdS action, the simplest LL gravity different from general relativity defined in $D \geq 5$ dimensions that includes a quadratic correction in the spacetime curvature given by the GB term,

$$
\begin{aligned}
I_{\mathrm{EGB}}= & \frac{1}{2 \kappa^{2}} \int d^{d+1} x \sqrt{-g} \\
& \times\left[R-2 \Lambda+\alpha\left(R^{2}-4 R_{\mu \nu} R^{\mu \nu}+R_{\mu \nu \lambda \sigma} R^{\mu \nu \lambda \sigma}\right)\right] .
\end{aligned}
$$

In the framework of String Theory, the GB term arises in the low-energy limit and the constant $\alpha$ is positive. In our case, we are rather concerned about restrictions imposed in a dual QFT, se we keep $\alpha$ an arbitrary real constant. The gravitational equations of motion in (5) becomes

$G_{v}^{\mu}+H_{v}^{\mu}=T_{v}^{\mu}$,

where we have introduced the Einstein tensor with the cosmological term,

$G_{v}^{\mu}=R_{v}^{\mu}-\frac{1}{2} \delta_{v}^{\mu} R+\Lambda \delta_{v}^{\mu}$

and the Lanczos tensor that describes contribution of the quadratic-curvature gravitational terms,

$$
\begin{aligned}
H_{v}^{\mu}= & -\frac{\alpha}{8} \delta_{\nu \nu_{1} \ldots \nu_{4}}^{\mu \mu_{1} \ldots \mu_{4}} R_{\mu_{1} \mu_{2}}^{\nu_{1} \nu_{2}} R_{\mu_{3} \mu_{4}}^{\nu_{3} \nu_{4}}, \\
= & -\frac{\alpha}{2} \delta_{v}^{\mu}\left(R^{2}-4 R^{\alpha \beta} R_{\alpha \beta}+R^{\alpha \beta \lambda \sigma} R_{\alpha \beta \lambda \sigma}\right) \\
& +2 \alpha\left(R R_{v}^{\mu}-2 R^{\mu \lambda} R_{\lambda \nu}-2 R_{\lambda \nu \sigma}^{\mu} R^{\lambda \sigma}+R^{\mu \alpha \lambda \sigma} R_{v \alpha \lambda \sigma}\right) .
\end{aligned}
$$

In EGB AdS gravity, second order polynomial (7) gives two (real) different radii square

$\ell_{\mathrm{eff}}^{( \pm) 2}=\frac{2 \alpha(d-2)(d-3)}{1 \pm \sqrt{1-\frac{4 \alpha}{\ell^{2}}(d-2)(d-3)}}$,

when $\alpha<\alpha_{\mathrm{CS}}=\frac{\ell^{2}}{4(d-2)(d-3)}$. Then the 1.h.s. of the gravitational equations of motion (9) can be factorized,

$$
\begin{gathered}
-\frac{\alpha}{8} \delta_{\nu \nu_{1} \ldots \nu_{4}}^{\mu \mu_{1} \ldots \mu_{4}}\left(R_{\mu_{1} \mu_{2}}^{v_{1} \nu_{2}}+\frac{1}{\ell_{\mathrm{eff}}^{(+) 2}} \delta_{\mu_{1} \mu_{2}}^{\nu_{1} \nu_{2}}\right) \\
\times\left(R_{\mu_{3} \mu_{4}}^{\nu_{3} v_{4}}+\frac{1}{\ell_{\mathrm{eff}}^{(-) 2}} \delta_{\mu_{3} \mu_{4}}^{\nu_{3} \nu_{4}}\right)=T_{v}^{\mu} .
\end{gathered}
$$


Note that only the negative branch with the radius $\ell_{\mathrm{eff}}^{(-)}$ ("stringy" vacuum) reduces to the bare AdS radius $\ell$ when $\alpha \rightarrow 0$, whereas $\ell_{\text {eff }}^{(+)}$is a new AdS vacuum typical for the EGB gravity. A linearized theory around the stringy vacuum shows the presence of the ghosts [65] indicating that this vacuum might be false, but the unstable modes are not excited by the spherically symmetric black hole $[66,67]$. Thus, because we are looking at the thermal CFTs dual to spherical black holes, we shall allow, in principle, both vacua in our discussion.

On the other hand, we shall omit the Chern-Simons point, $\alpha_{\mathrm{CS}}$, in the space of parameters, where the theory has the unique AdS radius $\left(\ell_{\mathrm{eff}}^{(-) 2}=\ell_{\mathrm{eff}}^{(+) 2}=\ell^{2} / 2\right)$ and the AdS vacuum is two-fold degenerate, because in that point the EGB Lagrangian becomes a Chern-Simons AdS gravity [68] that has augmented number of gauge symmetries and has to be treated independently [69].

To study black holes, we assume a static, maximally symmetric metric in the local coordinates $x^{\mu}=\left(t, r, y^{m}\right)$, $m=2, \ldots d$,

$$
\begin{aligned}
d s^{2}= & g_{\mu \nu}(x) d x^{\mu} d x^{\nu}=-f(r) d t^{2} \\
& +\frac{d r^{2}}{f(r) N(r)}+r^{2} \gamma_{m n}(y) d y^{m} d y^{n} .
\end{aligned}
$$

The radial coordinate is chosen so that the boundary is placed at radial infinity, $r \rightarrow \infty$, and parameterized by $x^{i}=\left(t, y^{m}\right)$. The metric $\gamma_{n m}$ of the transversal section $r=$ Const describes a $(d-1)$-dimensional space with the constant curvature $k=1,0$ or -1 , corresponding to spherical, flat or hyperbolic geometry, respectively. In particular, $k$ determines the geometry of the event horizon of the black hole, $r_{+}$, defined as the largest root of the equation $f\left(r_{+}\right)=0$. We are interested in the outer region only, $r \geq r_{+}$, where $f(r) \geq 0$. The function $N(r)>0$ is finite at the horizon.

In order to have flatter boundary that is suitable for a holographic description of a fluid, we shall restrict our study to planar $\operatorname{AdS}_{d+1}$ black holes with noncompact horizons, $k=0$, whose transversal section is $\mathbb{R}^{d-1}$ with the flat metric $\gamma_{m n}=\delta_{m n}$. We also require that the curvature is slowly varying, that is, the black hole horizon is big, $r_{+} \gg \ell_{\text {eff }}$.

Planar asymptotically AdS spacetimes have the metric functions that behave for large $r$ as,

$$
\begin{aligned}
f(r) & \rightarrow \frac{r^{2}}{\ell_{\mathrm{eff}}^{2}}+\mathcal{O}(1 / r), \\
f(r) N(r) & \rightarrow \frac{r^{2}}{\ell_{\mathrm{eff}}^{2}}+\mathcal{O}(1 / r),
\end{aligned}
$$

independently on how fast $\mathcal{O}(1 / r)$ tends to zero. The Hawking temperature of the black hole (14) in this spacetime reads

$$
T=\frac{1}{4 \pi} f^{\prime}\left(r_{+}\right) \sqrt{N\left(r_{+}\right)},
$$

and it increases linearly with $r_{+}$, so that the large horizon approximation corresponds to high temperatures in a holographically dual field theory.

We also assume that the black hole is electrically charged, with an Abelian gauge field that has the same isometries as the metric (14),

$A_{\mu}=\phi(r) \delta_{\mu}^{t}$

The field strength is expressed in terms of the electric field $E(r)=-\phi^{\prime}(r)$ as $F_{\mu \nu}=E(r) \delta_{\mu \nu}^{t r}$, where the prime denotes the radial derivative. Note that $F^{2}=-2 N E^{2}$.

The unknown functions $f(r), N(r), \phi(r)$ and $\Psi(r)$ obey the differential equations

$$
\begin{aligned}
& 0=\mathcal{E}_{\mu \nu}:=G_{\mu \nu}+H_{\mu \nu}-T_{\mu \nu}, \\
& 0=\mathcal{E}^{\mu}:=\frac{1}{\sqrt{-g}} \partial_{\nu}\left(4 \sqrt{-g} F^{\mu \nu} \frac{d \mathcal{L}}{d F^{2}}\right)-\mathcal{F} g^{\mu \nu} A_{\nu}, \\
& 0=\mathcal{E}:=\frac{1}{\sqrt{-g}} \partial_{\mu}\left(\sqrt{-g} g^{\mu \nu} \partial_{\nu} \Psi\right)-m^{2} \Psi-\frac{1}{2} \frac{d \mathcal{F}}{d \Psi} g^{\mu \nu} A_{\mu} A_{\nu} .
\end{aligned}
$$

For sufficiently high temperatures, these equations possess a solution without scalar field, $\Psi=0$, that is dual to a holographic QFT without condensate. Its most general form for an arbitrary GB coupling $\alpha$ and the NED Lagrangian $\mathcal{L}\left(F^{2}\right)$ was found in [70],

$$
\begin{aligned}
f(r)= & \frac{r^{2}}{2 \alpha(d-2)(d-3)} \\
& \times\left[1 \pm \sqrt{1-4 \alpha(d-2)(d-3)\left(\frac{1}{\ell^{2}}-\frac{\mu}{r^{d}}+\frac{2 \mathcal{T}(q, r)}{(d-1) r^{d}}\right)}\right],
\end{aligned}
$$

where $\mu$ is an integration constant related to the black hole mass, and the positive function $\mathcal{T}(q, r)$ corresponds to the total matter energy in the region between the horizon and the distance of radius $r$,

$\mathcal{T}(q, r)=\left.\frac{2}{d}\left(\frac{1}{4} r^{d} \mathcal{L}-q r E+(d-1) q \phi\right)\right|_{r_{+}} ^{r}$

When $\Psi=0$, the electric field is calculated from the algebraic equation

$\left.E \frac{d \mathcal{L}}{d F^{2}}\right|_{F^{2}=-2 E^{2}}=-\frac{q}{r^{d-1}}$.

In the special case of the Born-Infeld Lagrangian $\mathcal{L}\left(F^{2}\right)$ [71], the solution and its thermodynamics were discussed in [72].

When $\Psi \neq 0,(18)$ become a system of nonlinear differential equations. With help of the identities given in Appendix A, we write them in components as 


$$
\begin{aligned}
\mathcal{E}_{r}^{r}= & \frac{d-1}{2}\left[(d-2) \frac{N f}{r^{2}}+\frac{N f^{\prime}}{r}-2 \alpha\right. \\
& \left.\times(d-2)(d-3) N^{2}\left(\frac{f f^{\prime}}{r^{3}}+\frac{d-4}{2} \frac{f^{2}}{r^{4}}\right)-\frac{d}{\ell^{2}}\right]-T_{r}^{r}, \\
\mathcal{E}_{t}^{t}= & \mathcal{E}_{r}^{r}+\frac{N^{\prime} f}{2 r}(d-1)\left(1-2 \alpha(d-2)(d-3) \frac{N f}{r^{2}}\right) \\
& +\frac{1}{2} f N \Psi^{\prime 2}+\frac{\mathcal{F} \phi^{2}}{2 f},
\end{aligned}
$$

where the matter energy-momentum tensor contributes through

$T_{r}^{r}=\frac{1}{2} \mathcal{L}+2 N E^{2} \frac{d \mathcal{L}}{d F^{2}}+\frac{\mathcal{F} \phi^{2}}{4 f}-\frac{1}{4} m^{2} \Psi^{2}+\frac{1}{4} f N \Psi^{\prime 2}$.

One can also check that, as an effect of the backreaction of the gravitational field, the components $T_{t}^{t}$ and $T_{r}^{r}$ differ, that is,

$T_{t}^{t}=T_{r}^{r}-\frac{1}{2} f N \Psi^{\prime 2}-\frac{\mathcal{F} \phi^{2}}{2 f}$.

The presence of the scalar field decreases total matter energy density (because $f, N, \mathcal{F}>0$ outside the horizon). This means that, if a solution with the scalar field exists, then we can expect that it would decrease the total energy of the system and, therefore, be energetically more favorable, producing a phase transition.

The scalar field equation (18) in the chosen ansatz reads

$\mathcal{E}=\frac{\sqrt{N}}{r^{d-1}}\left(r^{d-1} \sqrt{N} f \Psi^{\prime}\right)^{\prime}-m^{2} \Psi+\frac{d \mathcal{F}}{d \Psi} \frac{\phi^{2}}{2 f}$.

In the NED equation in (18), only the time-like component is non-trivial,

$\mathcal{E}^{t}=-\frac{\sqrt{N}}{r^{d-1}}\left(4 r^{d-1} \sqrt{N} E \frac{d \mathcal{L}}{d F^{2}}\right)^{\prime}+\frac{\mathcal{F} \phi}{f}$.

We define the electric potential at distance $r$ measured with respect to the horizon $r_{+}$as

$\phi(r)=-\int_{r_{+}}^{r} d s E(s)$

The quantity of physical interest is the chemical potential $\Phi=\phi(\infty)-\phi\left(r_{+}\right)$, or the potential at infinity measured with respect to the event horizon,

$\Phi=\phi(\infty)=-\int_{r_{+}}^{\infty} d s E(s)$
This choice of the reference point satisfies $\phi\left(r_{+}\right)=0$, since any other referent point would lead to non-vanishing $\phi\left(r_{+}\right)$and a negative effective mass of the scalar field [12, 13]. Indeed, the electric potential couples to the scalar field so that, from (25), it contributes to its effective mass as $m_{\text {eff }}^{2}=$ $m^{2}-\left.\frac{\phi^{2}}{f} \frac{d \mathcal{F}}{d \Psi^{2}}\right|_{\Psi=0}$. This mass can be divergent on the horizon unless we impose $\phi=0$ there. It is also worthwhile noticing that the effective potential for the scalar field, leading to the equation of motion (25), has the form

$V_{\text {eff }}=\frac{1}{2} m^{2} \Psi^{2}-\frac{\phi^{2}}{2 f} \mathcal{F}(\Psi)$,

and because $\mathcal{F}$ is positive, the interaction decreases the effective potential and therefore the total energy of the system. The systems with unbounded potentials in gravity are, in general, known to lead to hairy black hole solutions.

Finally, it is straightforward to check that the gravitational equation $\mathcal{E}_{m}^{n}$ is not independent. Using the expressions (102) given in Appendix A, we find

$\mathcal{E}_{n}^{m}=\delta_{n}^{m}\left[\frac{\left(r^{d-1} \mathcal{E}_{r}^{r}\right)^{\prime}}{r^{d-2}}+\frac{r f^{\prime}}{2 f}\left(\mathcal{E}_{r}^{r}-\mathcal{E}_{t}^{t}\right)+\frac{r}{2}\left(\Psi^{\prime} \mathcal{E}-E \mathcal{E}^{t}\right)\right]$,

and therefore this equation always vanishes.

The field equations (22), (25), and (26) are at most second order differential equations in $\{f, N, \phi, \Psi\}$, defined between the horizon and the asymptotic boundary. Thus, for each field we have to impose at most two boundary conditions, at $r_{+}$ and $r \rightarrow \infty$. Asymptotic sector we shall discuss later. As respect to $r_{+}$, we require that all fields and their derivatives are finite on the horizon, as well as $f\left(r_{+}\right)=0, \phi\left(r_{+}\right)=0$ and $f^{\prime}\left(r_{+}\right)$fixed by the temperature. Then the values of other fields and their derivatives at $r_{+}$can easily be deduced from the equations of motion. The scalar field, for example, has to satisfy

$m^{2} \Psi\left(r_{+}\right)=4 \pi T \sqrt{N\left(r_{+}\right)} \Psi^{\prime}\left(r_{+}\right.$,

and $E\left(r_{+}\right)$and $N^{\prime}\left(r_{+}\right)$are obtained from (22) and (26).

For an arbitrary $r$, the field equations are not exactly soluble when $\psi \neq 0$. To deal with them analytically, one can use the matching method, that was first applied to superconductors with higher-order corrections in [73]. The method consists in finding two approximative solutions to the equations of motion in the leading order: one in the vicinity of the horizon and another in the asymptotic region. These two solutions are matched smoothly at the arbitrary intermediate point. This technique allows to obtain an analytic expression for the critical temperature. The results depend on the matching point parameter $z_{m}$, even though its presence does 
not change quantitatively the features of a phase transition. Another analytic method found in the literature is the SturmLiouville one, which was used to study holographic superconductors in [33].

In our approach, we shall use only an asymptotic expansion. When the gravity action is finite in the asymptotic region, its expansion in the vicinity of the AdS boundary can be viewed as a holographic reconstruction of the bulk fields (metric and matter fields) starting from the boundary field data [74]. This method cannot be used to fix all parameters in the expansion because there always remain some unfixed coefficients at a given order, depending on the dimension. This ambiguity is well understood in the Fefferman-Graham coordinate frame [75] as coming from non-local terms in the quantum effective action, precisely the ones related to the holographic stress tensor [76]. Even with this ambiguity, the subleading orders encode the information on the existence of phase transitions for a given set of coupling constants. When the transition exists, they enable one to calculate the critical temperature and the critical exponent in an analytic way.

In order to develop the above ideas in detail, first we have to remove the infrared divergences in the action. Then we shall construct the on-shell action that will yield, in the Euclidean section, the thermodynamic potential of the black hole that is identified with the free energy in holographic QFT. This result can be obtained exactly.

\section{Euclidean bulk action}

We evaluate the bulk action (4) using the equations of motion and show that it is divergent. The Euclidean action $I_{0}^{E}=$ $-i I_{0}=\int d^{d+1} x \sqrt{-g} \mathcal{L}_{0}$ is obtained by the Wick rotation of the time coordinate, $t=-i \tau$, where the Euclidean time $\tau$ is periodic, with the period $T^{-1}$, in order to avoid a conical singularity at the horizon. Assuming that the bulk Lagrangian $\mathcal{L}_{0}$, evaluated on-shell for a static and maximally symmetric solution, depends only on the radial coordinate, we obtain

$I_{0}^{E}=-\frac{V_{d-1}}{T} \int_{r_{+}}^{\infty} d r \frac{r^{d-1}}{\sqrt{N}} \mathcal{L}_{0}(r)$

where $V_{d-1}=\int d^{d-1} y \sqrt{\gamma}$ is the (infinite) volume of the flat transversal section. The physical quantity is an action per unit volume.

Using the gravitational equation of motion (22), the EGB term becomes

$$
\begin{aligned}
\mathcal{L}_{\mathrm{EGB}}= & -\frac{\sqrt{N}}{r^{d-1}} \\
& \times\left[\sqrt{N} f^{\prime}\left(r^{d-1}-2 \alpha(d-1)(d-2) r^{d-3} N f\right)\right]^{\prime}-2 T_{t}^{t} .
\end{aligned}
$$

Furthermore, the NED Lagrangian density, $\mathcal{L}$, and the scalar field Lagrangian density, $\mathcal{L}_{\mathrm{S}}$, can be calculated directly from the energy-momentum tensor (24),

$\mathcal{L}+\mathcal{L}_{\mathrm{S}}=2 T_{t}^{t}-4 N E^{2} \frac{d \mathcal{L}}{d F^{2}}+\frac{\mathcal{F} \phi^{2}}{f}$.

Summing up all contributions, the bulk Euclidean action becomes

$$
\begin{aligned}
I_{0}^{E}= & \frac{V_{d-1}}{2 \kappa^{2} T} \int_{r_{+}}^{\infty} d r\left[r^{d-1} \sqrt{N} f^{\prime}\left(1-2 \alpha(d-1)(d-2) \frac{N f}{r^{2}}\right)\right]^{\prime} \\
& +\frac{V_{d-1}}{2 \kappa^{2} T} \int_{r_{+}}^{\infty} d r r^{d-1}\left(4 \sqrt{N} E^{2} \frac{d \mathcal{L}}{d F^{2}}-\frac{\mathcal{F} \phi^{2}}{f \sqrt{N}}\right)
\end{aligned}
$$

where we omit writing that $\frac{d \mathcal{L}}{d F^{2}}$ is evaluated at $F^{2}=$ $-2 N E^{2}$. In order to show that the second line in the above equation is also a total derivative, we use the electromagnetic equation of motion ( 26) and write

$r^{d-1}\left(4 \sqrt{N} E^{2} \frac{d \mathcal{L}}{d F^{2}}-\frac{\mathcal{F} \phi^{2}}{f \sqrt{N}}\right)=-\left(4 r^{d-1} \sqrt{N} \phi E \frac{d \mathcal{L}}{d F^{2}}\right)^{\prime}$,

and we arrive at the final form of the bulk action,

$$
\begin{aligned}
I_{0}^{E}= & \frac{V_{d-1}}{2 \kappa^{2} T}\left[r^{d-1} \sqrt{N} f^{\prime}\left(1-2 \alpha(d-1)(d-2) \frac{N f}{r^{2}}\right)\right. \\
& \left.-4 r^{d-1} \sqrt{N} \phi E \frac{d \mathcal{L}}{d F^{2}}\right]\left.\right|_{r_{+}} ^{\infty} .
\end{aligned}
$$

This expression is valid for any charged black hole solution, independently on its explicit form, for all NED and scalar interactions $\mathcal{L}$ and $\mathcal{F}$, and any value of the GB constant $\alpha$.

Equation (37) is clearly divergent for the global AdS space, and therefore it is IR divergent in asymptotically AdS sector, and it has to be regularized by a suitable addition of boundary terms.

\section{Variational principle and boundary terms}

An action functional is well defined if it satisfies the finite action principle, that is, it is differentiable upon taking variational derivatives in the fields and free from IR divergences. This means that the action has to reach an extremum for a given set of boundary conditions. Removal of the divergences in the asymptotic region can be achieved if one supplements the boundary term $B$ to the bulk action $I_{0}$, so that the total 
action $I=I_{0}+B$ fulfills the above conditions. As a consequence, the finite total action also ensures the finiteness of the Noether charges and the Euclidean action, which is necessary for studying the black hole thermodynamics.

The pure gravitational part of the bulk action $I_{0}$ does not fulfill the above conditions. Indeed, the on-shell boundary terms obtained from the variation of (4) can be written, using the Stokes theorem in the spacetime with a boundary whose an outward-pointing unit normal is $n_{\mu}$, as

$$
\begin{aligned}
\delta I_{0}= & \frac{1}{2 \kappa^{2}} \int d^{d} x \sqrt{-h} n_{\mu} \\
& \times\left[-\delta_{\nu v_{1} \nu_{2} \nu_{3}}^{\mu \mu_{1} \mu_{2} \mu_{3}} g^{\nu \alpha} \delta \Gamma_{\mu_{1} \alpha}^{\nu_{1}}\left(\alpha R_{\mu_{2} \mu_{3}}^{\nu_{2} \nu_{3}}+\frac{1}{(d-1)(d-2)} \delta_{\mu_{2}}^{\nu_{2}} \delta_{\mu_{3}}^{\nu_{3}}\right)\right. \\
& \left.+\delta A_{\nu} 4 F^{\mu \nu} \frac{d \mathcal{L}}{d F^{2}}-\delta \Psi \nabla^{\mu} \Psi-\mathcal{F} \delta p\left(\nabla^{\mu} p-A^{\mu}\right)\right]
\end{aligned}
$$

In order to identify the boundary quantities in a simple way, it is convenient to choose the local coordinates as $x^{\mu}=$ $\left(r, x^{i}\right)$, where $x^{i}(i=2, \ldots d)$ parameterize the boundary placed at $r=$ Const, so that the normal vector has the form $n_{\mu}=(\mathcal{N}(r), 0)$. This choice of $n_{\mu}$ defines a Gauss-normal frame

$d s^{2}=\mathcal{N}^{2}(r) d r^{2}+h_{i j}(r, x) d x^{i} d x^{j}$,

where both the lapse function $\mathcal{N}(r)$ and the induced boundary metric $h_{i j}(r, x)$ can be related straightforwardly to the metric functions $f(r)$ and $N(r)$ used in the black hole ansatz (14),

$\mathcal{N}^{2}=\frac{1}{f N}, \quad h_{i j}=\left(\begin{array}{cc}-f & 0 \\ 0 & r^{2} \delta_{m n}\end{array}\right), \quad \sqrt{-h}=r^{d-1} \sqrt{f}$.

The extrinsic curvature of the boundary is defined as a covariant derivative of the unit normal, $K_{\mu \nu}=-\nabla_{\mu} n_{\nu}$. In a Riemann space it is symmetric, and in the ansatz (14) it has only non-vanishing components $K_{i j}$, or

$K_{j}^{i}=h^{i k} K_{k j}=-\frac{1}{2} \sqrt{N f} h^{i k} h_{k j}^{\prime}=\left(\begin{array}{cc}-\frac{f^{\prime}}{2} \sqrt{\frac{N}{f}} & 0 \\ 0 & -\frac{1}{r} \sqrt{N f} \delta_{n}^{m}\end{array}\right)$.

In the Gaussian frame, a variation of the Christoffel symbol gives rise to the terms proportional to both the variation of the induced metric, $\delta h_{i j}$, and the variation of the extrinsic curvature, $\delta K_{i j}$. This action, clearly, it is not differentiable, since $\delta I_{0} / \delta h_{i j}$ is not well defined. The differentiability of the gravitational action for the Dirichlet boundary conditions on the induced metric can be recovered by addition of the Gibbons-Hawking term. However, obtained Dirichlet action still remains IR divergent. To heal these divergences, one should apply one of known regularization methods suitable for application of the holographic principle, i.e., covariant and background independent. One possibility is to use the holographic renormalization [74,77], that is systematic, but technically involved procedure in higher dimensions, where a complete counterterm series has not been written explicitly yet.

We turn, therefore, to an alternative expression for a boundary term that regularizes gravity action, called Kounterterms because it depends explicitly on the extrinsic curvature $K_{i j}$. An advantage of this procedure is that the boundary term is well known for any LL gravity in any dimension because its form is universal, of a geometric origin [78,79]. Additionally, it is background independent, covariant and in Fefferman-Graham coordinates gives the results consistent with the holographic renormalization [80], in cases when the last one can be done explicitly.

The Kounterterms have different form in even and odd dimensions. In even dimensions $D=2 n>4$, the gravitational part of the boundary term corresponds to the $n$th Chern form in $d=2 n-1$ boundary dimensions,

$$
\begin{aligned}
B_{\mathrm{EGB}, 2 n-1}= & c_{2 n-1} \int d^{2 n-1} x \sqrt{-h} \\
& \times \int_{0}^{1} d u \delta_{i_{1} \ldots i_{2 n-1}}^{j_{1} \ldots j_{2 n-1}} K_{j_{1}}^{i_{1}} \Sigma_{j_{2} j_{3}}^{i_{2} i_{3}}(u) \ldots \Sigma_{j_{2 n-2} j_{2 n-1}}^{i_{2 n-2} i_{2 n-1}}(u),
\end{aligned}
$$

where the constant $c_{2 n-1}$ reads [81]

$c_{2 n-1}=-\frac{\left(-\ell_{\mathrm{eff}}^{2}\right)^{n-1}}{\kappa^{2}(2 n-2) !}\left(1-\frac{2 \alpha}{\ell_{\mathrm{eff}}^{2}}(2 n-2)(2 n-3)\right)$.

The tensor $\Sigma_{j_{1} j_{2}}^{i_{1} i_{2}}=\mathcal{R}_{j_{1} j_{2}}^{i_{1} i_{2}}-u^{2}\left(K_{j_{1}}^{i_{1}} K_{j_{2}}^{i_{2}}-K_{j_{2}}^{i_{1}} K_{j_{1}}^{i_{2}}\right)$ has symmetries of the Riemann tensor and it is constructed from the intrinsic boundary curvature $\mathcal{R}^{i}{ }_{j k l}(h)$ and the extrinsic curvature $K_{j}^{i}$. With the boundary term (42), the action principle for the gravitational fields is well posed if the spacetime is asymptotically locally AdS,

$R_{j_{1} j_{2}}^{i_{1} i_{2}}+\frac{1}{\ell_{\mathrm{eff}}^{2}} \delta_{j_{1} j_{2}}^{i_{1} i_{2}}=0, \quad$ on $\partial \mathcal{M}$.

For the flat transversal section, $\mathcal{R}^{i}{ }_{j k l}$ vanishes in $B_{\mathrm{EGB}, 2 n-1}$ and the parametric integral can be solved exactly,

$B_{\mathrm{EGB}, 2 n-1}=(-1)^{n-1}(2 n-2) ! c_{2 n-1} \int d^{2 n-1} x \sqrt{-h} \operatorname{det} K_{j}^{i}$. 
Using (40) and (41), the determinant of the extrinsic curvature is evaluated as

$\operatorname{det} K_{j}^{i}=\frac{1}{d !} \delta_{j_{1} \ldots j_{d}}^{i_{1} \ldots i_{d}} K_{i_{1}}^{j_{1}} \ldots K_{i_{d}}^{j_{d}}=-N^{n-\frac{1}{2}} \frac{f^{\prime} f^{n-\frac{3}{2}}}{2 r^{2 n-2}}$.

Similarly, the Euclidean gravitational boundary term in even dimensions, calculated in the chosen ansatz, reads

$B_{\mathrm{EGB}, 2 n-1}^{E}=\frac{V_{2 n-2}}{2 T}(-1)^{n}(2 n-2) ! c_{2 n-1} \lim _{r \rightarrow \infty}\left(N^{n-\frac{1}{2}} f^{n-1} f^{\prime}\right)$.

In odd dimensions $D=2 n+1$, a universal boundary term is derived from the second fundamental form, and its expression given in terms of a double parametric integration reads

$$
\begin{aligned}
B_{\mathrm{EGB}, 2 n}= & c_{2 n} \int^{2 n} x \sqrt{-h} \int_{0}^{1} d u \\
& \times \int_{0}^{u} d s \delta_{i_{1} \ldots i_{2 n}}^{j_{1} \ldots i_{2 n}} K_{j_{1}}^{i_{1}} \delta_{j_{2}}^{i_{2}} \Sigma_{j_{3} j_{4}}^{i_{3} i_{4}}(u, s) \ldots \Sigma_{j_{2 n-1} j_{2 n}}^{i_{2 n n} i_{2 n}}(u, s) .
\end{aligned}
$$

Now the matrix with the symmetries of the Riemann tensor is given by the expression $\Sigma_{j_{1} j_{2}}^{i_{1} i_{2}}=\mathcal{R}_{j_{1} j_{2}}^{i_{1} i_{2}}-$ $u^{2}\left(K_{j_{1}}^{i_{1}} K_{j_{2}}^{i_{2}}-K_{j_{2}}^{i_{1}} K_{j_{1}}^{i_{2}}\right)+\frac{s^{2}}{\ell_{\text {eff }}^{2}} \delta_{j_{1} j_{2}}^{i_{1} i_{2}}$, and the coefficient $c_{2 n}$ has the same as the one in Einstein-Hilbert AdS gravity [82], only the AdS radius is replaced by the effective one,

$c_{2 n}=-\frac{\left(-\ell_{\mathrm{eff}}^{2}\right)^{n-1}}{\kappa^{2} 2^{3 n-3}(n-1) !^{2}}\left(1-\frac{2 \alpha}{\ell_{\mathrm{eff}}^{2}}(2 n-1)(2 n-2)\right)$.

The action is stationary on-shell for asymptotically locally AdS spaces that satisfy $\delta K_{i j}=0$ on $\partial \mathcal{M}$ [81]. The last condition is equivalent to the Dirichlet boundary condition on the induced metric, as $K_{i j}$ and $h_{i j}$ are proportional in the leading order near the boundary.

Evaluated on the equations of motion, the Euclidean boundary term reads

$$
\begin{aligned}
B_{\mathrm{EGB}, 2 n}^{E}= & -\frac{V_{2 n-1}}{T n} 2^{n-2}(2 n-1) ! c_{2 n} \\
& \times \lim _{r \rightarrow \infty} \sqrt{N}\left[\left(f-\frac{r f^{\prime}}{2}\right)\left(-N f+\frac{r^{2}}{\ell_{\mathrm{eff}}^{2}}\right)^{n-1}\right. \\
& \left.+n r f^{\prime}(-N f)^{n-1}{ }_{2} F_{1}\left(1-n, \frac{1}{2} ; \frac{3}{2} ; \frac{r^{2}}{\ell_{\mathrm{eff}}^{2} N f}\right)\right],
\end{aligned}
$$

where the ordinary hypergeometric function ${ }_{2} F_{1}\left(1-n, \frac{1}{2}\right.$; $\frac{3}{2} ; \frac{r^{2}}{\ell_{\text {eff }}^{2} N f}$ ), represented by the hypergeometric series (see Appendix B), is just a compact way to write the counterterm series using the expansion

$$
\begin{aligned}
& { }_{2} F_{1}\left(1-n, \frac{1}{2} ; \frac{3}{2} ; \frac{r^{2}}{\ell_{\mathrm{eff}}^{2} N f}\right) \\
& \quad=1-\frac{n-1}{3} \frac{r^{2}}{\ell_{\mathrm{eff}}^{2} N f}+\frac{(n-1)(n-2)}{10} \frac{r^{4}}{\ell_{\mathrm{eff}}^{4} N^{2} f^{2}}+\cdots
\end{aligned}
$$

The above series converges when $\frac{r^{2}}{\ell_{\mathrm{eff}}^{2} N f}<1$.

Now we consider the Abelian gauge field and ask the action to be stationary under its variation. The NED surface term in (38) vanishes when the gauge field is held fixed on the boundary, $\delta A_{i}=0$. If the boundary condition is chosen in that way, the Euclidean action corresponds to an ensemble with fixed electric potential, $\phi$, and it is proportional to the Gibbs thermodynamic potential $G(T, \phi, \ldots)$ in grand canonical ensemble.

We shall, however, choose the boundary term of the form

$B_{\mathrm{NED}}=\frac{2}{\kappa^{2}} \int d^{d+1} x \partial_{\mu}\left(\sqrt{-g} A_{\nu} F^{\nu \mu} \frac{d \mathcal{L}}{d F^{2}}\right)$.

In this case, the on-shell variation of the total NED action becomes

$\delta\left(I_{\mathrm{NED}}+B_{\mathrm{NED}}\right)=-\frac{2}{\kappa^{2}} \int d^{d} x A_{\mu} \delta\left(\sqrt{-h} n_{\nu} F^{\nu \mu} \frac{d \mathcal{L}}{d F^{2}}\right)$.

When $h_{i j}$ is fixed on the boundary (the Dirichlet boundary conditions for the metric), the gauge field $A_{\mu}$ has to satisfy the Neumann-like boundary condition $\delta\left(F_{r i} \frac{d \mathcal{L}}{d F^{2}}\right)=0$ in order to give rise a stationary action. We will show in the next section that the electric charge $Q$ is proportional to the quantity $\sqrt{-h} n_{v} F^{v \mu} \frac{d \mathcal{L}}{d F^{2}}$ and, thus, keeping $F_{r i} \frac{d \mathcal{L}}{d F^{2}}$ fixed on $\partial \mathcal{M}$ describes a thermodynamic system whose electric charge is kept constant, $\delta Q=0$. In that case, the Euclidean action is related to the Helmholtz free energy $F(T, Q, \ldots)$ in the canonical ensemble. A choice of canonical or grand canonical ensemble is, therefore, governed by the choice of the boundary terms, because its addition to the Euclidean action is seen as the Legendre transformation $G=F-Q \phi$ of the corresponding thermodynamic potentials. A discussion of the choice of an electromagnetic boundary term in 4D Maxwell theory coupled to EH gravity and its application to black hole thermodynamics has been discussed in [83]. 
The on-shell evaluation of the Euclidean action of the boundary term (52) has the form

$B_{\mathrm{NED}}^{E}=\frac{2 V_{d-1}}{\kappa^{2} T} \lim _{r \rightarrow \infty}\left(r^{d-1} \phi \sqrt{N} E \frac{d \mathcal{L}}{d F^{2}}\right)$.

Finally, for the scalar field, the action is stationary when $\Psi$ and $p$ are held fixed on the boundary. We also note that the Euclidean action (37) does not have terms associated to scalar field, which means that there are no divergences associated to it either, and we can safely choose $B_{\mathrm{S}}=0$. Thus, we choose Dirichlet boundary conditions for the scalar field. Note that the vanishing of the scalar IR divergences is an effect of the gravitational backreaction. Namely, in the matter fields probe limit, when this backreaction is not taken into account, the scalar field becomes divergent and it requires additional counterterms (see, for example $[9,10]$ ). Our result that the backreaction heals the divergences is a particular feature of the chosen matter couplings-one should not expect the same to happen for, e.g., non-minimal interaction between $\Psi$ and the EM field, such as $\sigma(\Psi) \mathcal{L}\left(F^{2}\right)$.

In the next section, we use the Noether theorem to calculate conserved quantities associated to the local symmetries in the theory.

\section{Conserved quantities}

The action is invariant under Abelian gauge transformations that act non-trivially on the following fields:

$\delta_{\lambda} A_{\mu}=\partial_{\mu} \lambda, \quad \delta_{\lambda} p=\lambda$,

and whose associated Noether current reads

$J^{\mu}(\lambda)=\partial_{\nu}\left(\frac{\sqrt{-g}}{2 \kappa^{2}} \lambda 4 F^{\mu \nu} \frac{d \mathcal{L}}{d F^{2}}\right)$.

The charge density in the radial foliation is described by the component $J^{r}(\lambda)=\partial_{i}\left(\frac{\sqrt{-g}}{2 \kappa^{2}} \lambda 4 F^{r i} \frac{d \mathcal{L}}{d F^{2}}\right)$. Applying the Stokes theorem to the boundary manifold at $r \rightarrow \infty$ with the metric $h_{i j}$ whose time-slice boundary $t=$ Const, denoted by $\Sigma_{\infty}$, is defined by an outward-pointing time-like unit normal $u_{i}=\left(u_{t}, u_{m}\right)=(-\sqrt{f}, 0)$, we obtain a general formula for the total electric charge of the black hole,

$Q=-\frac{2 V_{d-1}}{\kappa^{2}} \lim _{r \rightarrow \infty}\left(r^{d-1} \sqrt{N} E \frac{d \mathcal{L}}{d F^{2}}\right)$.

This form of the charge justifies the interpretation of the boundary condition $\delta\left(F_{r i} \frac{d \mathcal{L}}{d F^{2}}\right)=0$ mentioned in the previous section as having $Q$ fixed.
Energy-momentum of the black hole is associated to the time-like diffeomorphisms $\xi=\xi^{\mu} \partial_{\mu}$ that act on the fields as Lie derivatives,

$£_{\xi} g_{\mu \nu}=\nabla_{\mu} \xi_{\nu}+\nabla_{\nu} \xi_{\mu}, \quad £_{\xi} \Psi=\xi^{\nu} \partial_{\nu} \Psi$,

$£_{\xi} A_{\mu}=\partial_{\mu}\left(\xi^{\nu} A_{\nu}\right)-\xi^{\nu} F_{\mu \nu}, \quad £_{\xi} p=\xi^{\nu} \partial_{\nu} p$.

The gravitational contribution to the conserved charges in EGB gravity coupled to NED, where the same regularization method is used, is calculated in [70] as

$Q[\xi]=\int_{\Sigma_{\infty}} d^{d-1} y \sqrt{\operatorname{det}\left(g_{m n}\right)} u_{j} \xi^{i} q_{i}^{j}$,

where $\xi$ is an asymptotic Killing vector. The charge density tensor $q_{i}^{j}(r)$ has different form in even and odd dimensions, depending on the boundary terms. In our case, $\xi=\partial_{t}$ and $g_{m n}=r^{2} \delta_{m n}$, so that the total energy is

$Q\left[\partial_{t}\right]=-V_{d-1} \lim _{r \rightarrow \infty} r^{d-1} \sqrt{f} q_{t}^{t}$.

In even dimensions ( $D=2 n$ ), the charge density tensor is given by the formula [70]

$$
\begin{aligned}
\left(q_{2 n}\right)_{i}^{j}= & \frac{1}{2 \kappa^{2}(2 n-2) ! 2^{n-2}} \delta_{i_{1} i_{2} \ldots i_{2 n-1}}^{j j_{2} \ldots j_{2 n-1}} K_{i}^{i_{1}} \\
& \times\left[\left(\delta_{j_{2} j_{3}}^{i_{2} i_{3}}+2 \alpha(2 n-2)(2 n-3) R_{j_{2} j_{3}}^{i_{2} i_{3}}\right)\right. \\
& \times \delta_{j_{4} j_{5}}^{i_{4} i_{5}} \ldots \delta_{j_{2 n-2} j_{2 n-1}}^{i_{2 n-2 i_{2 n-1}}} \\
& \left.+\kappa^{2}(2 n-2) ! c_{2 n-1} R_{j_{2} j_{3}}^{i_{2} i_{3}} \ldots R_{j_{2 n-2} j_{2 n-1}}^{i_{2 n-2} i_{2 n-1}}\right]
\end{aligned}
$$

where the Gauss-Codazzi relation $R_{k l}^{i j}=\mathcal{R}_{k l}^{i j}-K_{k}^{i} K_{l}^{j}+$ $K_{l}^{i} K_{k}^{j}$ has to be used in order to express $q_{i}^{j}$ completely in terms of the boundary quantities. Evaluating the above tensor in the ansatz (40) and (41), the total energy becomes

$$
\begin{aligned}
Q_{2 n}\left[\partial_{t}\right]= & \frac{V_{2 n-2}}{2 \kappa^{2}} \lim _{r \rightarrow \infty} r^{2 n-2} \sqrt{N} f^{\prime} \\
& \times\left[1-2 \alpha(2 n-2)(2 n-3) \frac{N f}{r^{2}}\right. \\
& \left.+\kappa^{2}(2 n-2) ! c_{2 n-1}\left(-\frac{N f}{r^{2}}\right)^{n-1}\right] .
\end{aligned}
$$

On the other hand, in odd dimensions $(D=2 n+1)$, the charge density tensor contains the following terms [70]:

$$
\begin{aligned}
q_{i}^{j}= & \left(q_{\mathrm{vac}}\right)_{i}^{j}+\frac{1}{2 \kappa^{2}(2 n-1) ! 2^{n-2}} \delta_{i_{1} \ldots i_{2 n}}^{j j_{2} \ldots j_{2 n}} K_{i}^{i_{1}} \delta_{j_{2}}^{i_{2}} \\
& \times\left[\left(\delta_{j_{3} j_{4}}^{i_{3} i_{4}}+2 \alpha(2 n-1)(2 n-2) R_{j_{3} j_{4}}^{i_{3} i_{4}}\right) \delta_{j_{5} j_{6}}^{i_{5} i_{6}} \ldots \delta_{j_{2 n-1} j_{2 n}}^{i_{2 n-1} i_{2 n}}\right.
\end{aligned}
$$




$$
\begin{aligned}
& +2 \kappa^{2}(2 n-1) ! n c_{2 n} \int_{0}^{1} d u\left(R_{j_{3} j_{4}}^{i_{3} i_{4}}+\frac{u^{2}}{\ell_{\mathrm{eff}}^{2}} \delta_{j_{3} j_{4}}^{i_{3} i_{4}}\right) \\
& \left.\times \ldots\left(R_{j_{2 n-1} j_{2 n}}^{i_{2 n-1} i_{2 n}}+\frac{u^{2}}{\ell_{\mathrm{eff}}^{2}} \delta_{j_{2 n-1} j_{2 n}}^{i_{2 n-1} i_{2 n}}\right)\right],
\end{aligned}
$$

where

$$
\begin{aligned}
\left(q_{\mathrm{vac}}\right)_{i}^{j}= & 2^{n-2} c_{2 n} \int_{0}^{1} d u u \delta_{k i_{2} \ldots i_{2 n}}^{j j_{2} \ldots j_{2 n}}\left(K_{i}^{k} \delta_{j_{2}}^{i_{2}}+K_{j_{2}}^{k} \delta_{i}^{i_{2}}\right) \\
& \times\left(\frac{1}{2} \mathcal{R}_{j_{3} j_{4}}^{i_{3} i_{4}}-u^{2} K_{j_{3}}^{i_{3}} K_{j_{4}}^{i_{4}}+\frac{u^{2}}{\ell_{\mathrm{eff}}^{2}} \delta_{j_{3}}^{i_{3}} \delta_{j_{4}}^{i_{4}}\right) \times \cdots \\
& \times\left(\frac{1}{2} \mathcal{R}_{j_{2 n-1} j_{2 n}}^{i_{2 n-1} i_{2 n}}-u^{2} K_{j_{2 n-1}}^{i_{2 n-1}} K_{j_{2 n}}^{i_{2 n}}+\frac{u^{2}}{\ell_{\mathrm{eff}}^{2}} \delta_{j_{2 n-1}}^{i_{2 n-1}} \delta_{j_{2 n}}^{i_{2 n}}\right)
\end{aligned}
$$

and again we have to use the Gauss-Codazzi relation for $R_{k l}^{i j}$ to express the tensor in terms of the boundary quantities. Note that the charge density does not vanish identically for global AdS, $R_{j_{2} j_{3}}^{i_{2} i_{3}}=-\frac{1}{\ell_{\mathrm{eff}}^{2}} \delta_{j_{2} j_{3}}^{i_{2} i_{3}}$, because the contribution $\left(q_{\mathrm{vac}}\right)_{i}^{j}$ accounts for the vacuum energy of AdS space. The vacuum energy depends only on the properties of the "empty" space, that is, the topological parameter $k$, the effective AdS radius and the gravitational couplings $\kappa^{2}, \alpha$; thus the presence of the scalar field does not influence on it. The formula for the vacuum energy of GB black holes in asymptotically AdS space was evaluated in [81], but because in our case the topological parameter is zero $(k=0)$, it vanishes. This can be seen explicitly. Evaluating $q_{t}^{t}$ in our ansatz, we obtain

$$
\begin{aligned}
q_{t}^{t}= & \left(q_{\mathrm{vac}}\right)_{t}^{t}+\frac{K_{t}^{t}}{\kappa^{2}}[1-2 \alpha(2 n-1)(2 n-2) \\
& \left.\times \frac{N f}{r^{2}}+2 \kappa^{2}(2 n-1) ! 2^{n-2} c_{2 n} \int_{0}^{1} d u\left(-\frac{N f}{r^{2}}+\frac{u^{2}}{\ell_{\mathrm{eff}}^{2}}\right)^{n-1}\right],
\end{aligned}
$$

where the integral can be expressed in terms of the hypergeometric function, as explained in Appendix B. Now we can show that the vacuum energy,

$\left(q_{\mathrm{vac}}\right)_{t}^{t}=\frac{2^{n-2}}{n}(2 n-1) ! c_{2 n} \sqrt{\frac{N}{f}}\left(\frac{f}{r}-\frac{f^{\prime}}{2}\right)\left(-\frac{N f}{r^{2}}+\frac{1}{\ell_{\mathrm{eff}}^{2}}\right)^{n-1}$,

does not contribute to the total energy $Q\left[\partial_{t}\right]$. For planar black holes in asymptotically AdS spacetime, the metric functions $f$ and $N$ satisfy the conditions (15). Evaluating the limit of $\left(q_{\mathrm{vac}}\right)_{t}^{t}$ in (60), we get

$$
\begin{aligned}
E_{\mathrm{vac}}= & -V_{2 n-1} \frac{2^{n-2}}{n}(2 n-1) ! c_{2 n} \lim _{r \rightarrow \infty} \sqrt{N} \\
& \times\left(f-\frac{r f^{\prime}}{2}\right)\left(-N f+\frac{r^{2}}{\ell_{\mathrm{eff}}^{2}}\right)^{n-1}=0,
\end{aligned}
$$

as expected. Thus, what remains is

$$
\begin{aligned}
Q_{2 n+1}\left[\partial_{t}\right]= & -\frac{V_{2 n-1}}{\kappa^{2}} \lim _{r \rightarrow \infty} r^{2 n-1} \sqrt{f} K_{t}^{t} \\
& \times\left[1-2 \alpha(2 n-1)(2 n-2) \frac{N f}{r^{2}}\right. \\
& +2 \kappa^{2}(2 n-1) ! 2^{n-2} c_{2 n}\left(-\frac{N f}{r^{2}}\right)^{n-1} \\
& \left.\times{ }_{2} F_{1}\left(1-n, \frac{1}{2} ; \frac{3}{2} ; \frac{r^{2}}{\ell_{\mathrm{eff}}^{2} N f}\right)\right] .
\end{aligned}
$$

In both even and odd dimensions $D$, total energy corresponds to the black hole mass that can be written as

$M=\frac{V_{D-2}}{2 \kappa^{2}} \lim _{r \rightarrow \infty} \mathcal{M}_{D}(r)$

with the function of radial coordinate given in even dimensions by

$$
\begin{aligned}
\mathcal{M}_{2 n}(r)= & r^{2 n-2} \sqrt{N} f^{\prime}[1-2 \alpha(2 n-2) \\
& \left.\times(2 n-3) \frac{N f}{r^{2}}+\kappa^{2}(2 n-2) ! c_{2 n-1}\left(-\frac{N f}{r^{2}}\right)^{n-1}\right],
\end{aligned}
$$

and in odd dimensions as

$$
\begin{aligned}
\mathcal{M}_{2 n+1}(r)= & \sqrt{N} f^{\prime}\left[r^{2 n-1}\left(1-2 \alpha(2 n-1)(2 n-2) \frac{N f}{r^{2}}\right)\right. \\
& +2 \kappa^{2}(2 n-1) ! 2^{n-2} c_{2 n} r(-N f)^{n-1} \\
& \left.\times{ }_{2} F_{1}\left(1-n, \frac{1}{2} ; \frac{3}{2} ; \frac{r^{2}}{\ell_{\text {eff }}^{2} N f}\right)\right] .
\end{aligned}
$$

Without scalar field, $N=1$ and the above formulas match the ones of [70].

It is straightforward to check that scalar and electromagnetic fields do not contribute to the mass. We shall show explicitly that, in the Euclidean section, addition of the boundary term $B_{\mathrm{NED}}^{E}$ performs a Legendre transformation of the thermodynamic potential. On the other hand, the scalar field does not contribute to the mass because the time-like isometry leaves the static scalar fields invariant, $£_{\partial_{t}} \Psi=0$ and $£_{\partial_{t}} p=0$, leading to the given result. Furthermore, we do not need the counterterms for $\Psi$. Thus, the above formula is 
the final, exact expression for the total energy of black holes with hair in nonlinear GB AdS gravity interacting with the St ückelberg scalar and NED field.

Now we proceed to evaluate the free energy for a chosen class of solutions.

\section{Quantum statistical relation for a GB superconductor}

Using the nomenclature of [84], quantum statistical relation is an expression for a thermodynamic potential of the system held at fixed temperature obtained using the quantum statistical mechanics, that is, directly from the partition function defined as exponent of the Euclidean action. The charges within it are the Noether ones, a consequence of the symmetries of the action, and they are expected to match the thermodynamic charges that appear in the first law of thermodynamics. This formula differs from the Smarr-like relation stemming from integration of the first law.

In order to find the statistical relation, we have to calculate the Euclidean action. Adding the contributions of the boundary terms (47) and (54) to the bulk action (37), the electromagnetic part cancels out at infinity and the total Euclidean action in even dimensions reads

$$
\begin{aligned}
I^{E}= & \frac{V_{2 n-2}}{2 \kappa^{2} T} \lim _{r \rightarrow \infty} \mathcal{M}_{2 n}(r) \\
& -\frac{V_{d-1}}{2 \kappa^{2} T}\left[\sqrt{N} f^{\prime}\left(r^{d-1}-2 \alpha(d-1)(d-2) r^{d-3} N f\right)\right. \\
& \left.-4 r^{d-1} \sqrt{N} \phi E \frac{d \mathcal{L}}{d F^{2}}\right]\left.\right|_{r=r_{+}}
\end{aligned}
$$

where we recognized the radial function $\mathcal{M}_{2 n}(r)$ from (70). The terms in the second line do not depend on the parity of the dimension $d$. On the horizon, the functions $f$ and $\phi$ vanish and $N$ and $E \frac{d \mathcal{L}}{d F^{2}}$ are finite, so using the definition of the Hawking temperature (16) and the black hole mass (69), we can write the Euclidean action as

$I^{E}=T^{-1} M-S$,

where the entropy $S=-\left.\frac{V_{d-1}}{T}\left[r^{d-1} \sqrt{f}\left(q-q_{\mathrm{vac}}\right)_{t}^{t}\right]\right|_{r_{+}}$is, as usual, the Noether charge on the horizon, giving

$S=\frac{2 \pi V_{d-1} r_{+}^{d-1}}{\kappa^{2}}$.

Note that the GB term does not contribute to the entropy when the black hole is planar. Additionally, the Stückelberg scalar does not modify the area law, as it happens for a conformally coupled scalar field $f(\Psi) R$ or other non-minimal interactions between $\Psi$ and curvature invariants [85].
A similar expression for the Euclidean action is found in odd dimensions, as well. Summing up (37), (50), and (54) and recognizing the expression for the mass (71) and vacuum energy (67), we obtain the total black hole energy, instead of the mass only,

$I^{E}=T^{-1}\left(M+E_{\mathrm{vac}}\right)-S$,

which finally reduces to (73) because $E_{\mathrm{vac}}=0$. The quantum statistical relation in canonical ensemble implies that the Helmholtz free energy, $F=T I^{E}$, has the correct form of the Legendre transformation of the internal energy,

$F=M-T S=\frac{V_{d-1}}{2 \kappa^{2}} \lim _{r \rightarrow \infty} \mathcal{M}_{D}(r)-\frac{2 \pi T V_{d-1} r_{+}^{d-1}}{\kappa^{2}}$

Thus, we obtain the exact expression for the thermodynamic potential of the family of black holes in EGB gravities that interact non-minimally with matter. The functions $\mathcal{L}$ and $\mathcal{F}$ do not enter this formula explicitly, but through the coupling constants.

\section{Free energy of a Lovelock superconductor in canonical and grand canonical ensemble}

The result for the free energy (76) can be generalized to any hairy LL AdS black hole coupled to NED and Stückelberg field. As shown in [78,79], the LL AdS action is regularized by the same boundary term as the one in Einstein-Hilbert or EGB gravity with negative cosmological constant in even or odd dimensions, which is known to be universal for any Lovelock gravity. The only quantity that changes is the coefficient $c_{d}$, which depends on $\ell_{\text {eff }}$ as one of the roots of the polynomial (7). Proceeding in the same way as in the EGB case, the Lovelock action evaluated on the black hole ansatz has the form

$$
\begin{aligned}
I_{\mathrm{LL}}= & \frac{1}{2 \kappa^{2}} \int d^{D} x \sum_{p=0}^{[d / 2]} \alpha_{p} \frac{r^{d-1}}{\sqrt{N}} \frac{(d-1) !}{(d-2 p+1) !} \frac{N}{r^{2}}\left(-\frac{f N}{r^{2}}\right)^{p-2} \\
& \times\left[\frac{p N^{\prime}}{2}\left((2 p-1) f f^{\prime}+2(d-2 p+1) \frac{f^{2}}{r}\right)\right. \\
& +N\left(p f f^{\prime \prime}+p(p-1) f^{\prime 2}+2 p(d-2 p+1) \frac{f f^{\prime}}{r}\right. \\
& \left.\left.+(d-2 p+1)(d-2 p) \frac{f^{2}}{r^{2}}\right)\right] .
\end{aligned}
$$

Compared with the dynamics of the EGB gravity, when the higher-order curvature corrections are added to the action, only the gravitational equations of motion change, 


$$
\begin{aligned}
\mathcal{E}_{r}^{r}= & \sum_{p=0}^{[d / 2]} \alpha_{p} \frac{(d-1) !}{2(d-2 p) !}\left(-\frac{f N}{r^{2}}\right)^{p-1} \\
& \times\left(p \frac{f^{\prime} N}{r}+(d-2 p) \frac{f N}{r^{2}}\right)-T_{r}^{r} \\
\mathcal{E}_{t}^{t}= & \mathcal{E}_{r}^{r}+\sum_{p=1}^{[d / 2]} \alpha_{p} \frac{p(d-1) !}{2(d-2 p) !}\left(-\frac{f N}{r^{2}}\right)^{p-1} \\
& \times \frac{f N^{\prime}}{r}+\frac{1}{2} f N \Psi^{\prime 2}+\frac{\mathcal{F} \phi^{2}}{2 f}
\end{aligned}
$$

and they reduce to (22) in EGB case. Using these equations, the total bulk action coupled to the matter fields generalizes (37) to

$$
\begin{aligned}
I_{0}^{E}= & \frac{V_{d-1}}{2 \kappa^{2} T}\left[r^{d-1} \sqrt{N} f^{\prime} \sum_{p=1}^{[d / 2]} \alpha_{p} \frac{p(d-1) !}{(d-2 p+1) !}\right. \\
& \left.\times\left(-\frac{f N}{r^{2}}\right)^{p-1}-4 r^{d-1} \sqrt{N} \phi E \frac{d \mathcal{L}}{d F^{2}}\right]\left.\right|_{r_{+}} ^{\infty} .
\end{aligned}
$$

Now we follow the steps of Sects. 5-7 in order to show that the mass has the form (69).

In even dimensions, the boundary term is given by the formula (42), where the Lovelock parameters $\alpha_{p}$ are introduced as

$$
\begin{aligned}
& \left(\delta_{j_{2} j_{3}}^{i_{2} i_{3}}+2 \alpha(2 n-2)(2 n-3) R_{j_{2} j_{3}}^{i_{2} i_{3}}\right) \delta_{j_{4} j_{5}}^{i_{4} i_{5}} \cdots \delta_{j_{2 n-2} j_{2 n-1}}^{i_{2 n-2} i_{2 n-1}}+\cdots \\
& =\sum_{p=1}^{n} \alpha_{p} \frac{p(2 n-2) !}{(2 n-2 p) !} R_{j_{2} j_{3}}^{i_{2} i_{3}} \ldots R_{j_{2} p-2 j_{2 p-1}}^{i_{2 p-2} i_{2 p-1}} \delta_{j_{2 p} j_{2 p+1}}^{i_{2 p} i_{2 p+1}} \ldots \delta_{j_{2 n-1} j_{2 n-1}}^{i_{2 n-2} i_{2 n-1}},
\end{aligned}
$$

and the effective AdS radius in $c_{2 n-1}$ is the one for the LL theory. After a straightforward calculation, one arrives at a radial function that defines the black hole mass in even dimensions,

$$
\begin{aligned}
\mathcal{M}_{2 n}(r)= & r^{2 n-2} \sqrt{N} f^{\prime}\left[\sum_{p=1}^{n-1} \alpha_{p} \frac{p(2 n-2) !}{(2 n-2 p) !}\left(-\frac{N f}{r^{2}}\right)^{p-1}\right. \\
& \left.+\kappa^{2}(2 n-2) ! c_{2 n-1}\left(-\frac{N f}{r^{2}}\right)^{n-1}\right]
\end{aligned}
$$

In odd dimensions, the boundary term has the form (48) with the following generalization:

$$
\begin{aligned}
& \left(\delta_{j_{3} j_{4}}^{i_{3} i_{4}}+2 \alpha(2 n-1)(2 n-2) R_{j_{3} j_{4}}^{i_{3} i_{4}}\right) \delta_{j_{5} j_{6}}^{i_{5} i_{6}} \cdots \delta_{j_{2 n-1} j_{2 n}}^{i_{2 n-1} i_{2 n}}+\cdots \\
& =\sum_{p=1}^{n} \alpha_{p} \frac{p(2 n-1) !}{(2 n-2 p+1) !} R_{j_{3} j_{4}}^{i_{3} i_{4}} \ldots R_{j_{2 p-1} j_{2 p}}^{i_{2 p-1} i_{2 p}} \delta_{j_{2 p+1} j_{2 p+2}}^{i_{2 p+1} i_{2 p+2}} \ldots \delta_{j_{2 n-1} j_{2 n}}^{i_{2 n-1} i_{2 n}},
\end{aligned}
$$

and using the AdS radius that depend on all LL coupling constants. The vacuum energy vanishes for the planar solutions, $E_{\mathrm{vac}}=0$, and the radial function that determines the black hole mass is

$$
\begin{aligned}
\mathcal{M}_{2 n+1}(r)= & \sqrt{N} f^{\prime}\left[r^{2 n-1} \sum_{p=1}^{n} \alpha_{p} \frac{p(2 n-1) !}{(2 n-2 p+1) !}\left(-\frac{N f}{r^{2}}\right)^{p-1}\right. \\
& +\kappa^{2}(2 n-1) ! 2^{n-1} c_{2 n} r(-N f)^{n-1} \\
& \left.\times{ }_{2} F_{1}\left(1-n, \frac{1}{2} ; \frac{3}{2} ; \frac{r^{2}}{\ell_{\mathrm{eff}}^{2} N f}\right)\right]
\end{aligned}
$$

The Helmholtz free energy obtained from the quantum statistical relation has the usual form,

$F=\frac{V_{d-1}}{2 \kappa^{2}} \lim _{r \rightarrow \infty} \mathcal{M}_{D}(r)-\frac{2 \pi T V_{d-1} r_{+}^{d-1}}{\kappa^{2}}$

At this point, it is straightforward to write an analogous result in the grand canonical ensemble. Let us recall once more that the previous results, calculated in the canonical ensemble, are obtained from the action that fulfills the boundary conditions $\delta I_{\text {can }}=0$ when $T, Q$ and $\Psi$ are held fixed on the boundary, and the on-shell Euclidean action becomes $I_{\text {can }}^{E}=T^{-1} F=T^{-1} M-S$.

On the other hand, in grand canonical ensemble, the action is stationary for the boundary conditions $\delta I_{\text {grand can }}=0$ when $T, \phi$ and $\Psi$ are held fixed on the boundary which, in practice, is realized by not adding the NED boundary term (52) to the bulk action. As explained in Sect. 5, its Euclidean continuation is related to the Gibbs potential $G$ as

$I_{\text {grand can }}^{E}=T^{-1} G=T^{-1} M-S-T^{-1} Q \Phi$,

which is exactly a Legendre transformation of the Helmholtz free energy. Here the mass has the same form as before and the conjugated variables $\Phi$ and $Q$ are [see Eqs. (28) and (57)],

$\Phi=\lim _{r \rightarrow \infty} \phi(r), \quad Q=-\frac{2 V_{d-1}}{\kappa^{2}} \lim _{r \rightarrow \infty}\left(r^{d-1} \sqrt{N} E \frac{d \mathcal{L}}{d F^{2}}\right)$.

Without scalar field, the above results coincide with the ones found in [86,87] for the EGB case.

It is worthwhile mentioning that all presented results can be generalized to topological black holes with non-flat transversal section $(k \neq 0)$, in the case one is interested in the study of the effects of the horizon topology to the properties of superconductors [88]. 


\section{Discussion: from the free energy to the phase transition}

The Helmholz free energy formula for LL AdS (84), which includes backreaction of the gravitational fields, contains all thermodynamic information about the holographic quantum system described by the partition function $Z=\mathrm{e}^{-F / T}$. The theory depends on a large number of parameters [up to $[d / 2]$ Lovelock gravity parameters, electromagnetic coupling constants contained in the function $\mathcal{L}\left(F^{2}\right)$ and the scalar couplings in $\mathcal{F}(\Psi)$ ]. Phase transitions will occur only for some values of these parameters. In the present literature, their values are chosen arbitrarily, in the points of the parameter space known that they would have a phase transition.

We address a different question, focused on obtaining a criterion about the parameters choice and, using it, a classification of all possible LL superconductors. This would enable theoretical design of a superconductor with the desired features through the choice of the coupling constants. In that way, the diversity of high- $T_{\mathrm{c}}$ superconductors would be directly related to the diversity of dual gravitational theories.

As the first step, we have to analyze the local and global minima of the free energy using the renormalized formula (84) and detect all possible phase transitions through the discontinuities in the free energy, similarly to an analysis in the Landau-Ginzburg description of superconductivity.

In $d$-dimensional thermal QFT, the temperatures are high. Since the gauge/gravity duality prescription relates $T$ to the Hawking temperature proportional to the horizon area, high $T$ corresponds to large black hole radius $r_{+}$. More precisely, in the gravity side, we have to require that the black hole horizon is big, $r_{+} \gg \ell_{\text {eff }}$, which we can also take as the first approximation in analytic calculations.

Thus, to obtain an initial information about possible phase transitions - that is, to detect development of hair in the black hole due to presence of the scalar field- we need only an asymptotic solution, but calculated in the subleading order. This enables to identify the point of the phase transition and also calculate its critical exponent $\beta$ and the critical temperature. Once there is a classification of the superconductors, we can also calculate the transport coefficients (superconductivity, energy gap, etc.), where further knowledge on the behavior of the solution away from the asymptotic region is also required.

To be more precise, let us focus again to the EGB AdS gravity with the matter. We have to power-expand in $\ell_{\text {eff }} / r<<1$ all unknown functions $f(r), N(r), \Psi(r), \phi(r)$ and all given functions $\mathcal{F}(\Psi(r)), \mathcal{L}\left(F^{2}(r)\right)$. Note that $\mathcal{F}$ and $\mathcal{L}$ can be chosen arbitrarily, as they define interactions between the gravity and matter fields, whereas $f, N, \Psi$ and $\phi$ should be uniquely determined for given boundary conditions set. Thus, assuming the known asymptotic behavior, any function $X(r)$ can be expanded in powers of $1 / r$, where the constants $\ell_{\text {eff }}$ are absorbed in the coefficients $X_{n}$ for the sake of simplicity. We numerate only non-vanishing coefficients $X_{n}$ with $n \geq 0$.

In general, we are interested in generic black holes, that is, the ones where (i) the equation of motion for the field $X(r)$ uniquely determines the coefficient $X_{n}$ in the $n$th order of the asymptotic power expansion in terms of the leading order, and (ii) there are no anomalies, that is, no logarithmic terms in the expansion are present.

There will also be other particular solutions [that do not fulfill the condition (i)] that have to be considered separately. The anomalies [that do not satisfy (ii)] are of particular interest and they will be addressed somewhere else.

For an asymptotically AdS spacetime that satisfies (15), we seek a solution in the form

$$
\begin{aligned}
f(r) & =\frac{r^{2}}{\ell_{\mathrm{eff}}^{2}}\left(1+\frac{f_{0}}{r^{u}}+\frac{f_{1}}{r^{u_{1}}}+\cdots\right), \\
N(r) & =1+\frac{N_{1}}{r^{s_{1}}}+\cdots, \\
\Psi(r) & =\frac{\Psi_{0}}{r^{\Delta}}+\frac{\Psi_{1}}{r^{\Delta_{1}}}+\cdots, \\
\phi(r) & =\Phi+\frac{\phi_{0}}{r^{\lambda}}+\frac{\phi_{1}}{r^{\lambda_{1}}}+\cdots,
\end{aligned}
$$

where $0<u<u_{n}<u_{n+1}$ and similarly for all other power factors. The interaction is given in terms of the set of parameters that determine its fall-off,

$$
\begin{aligned}
\mathcal{F}(\Psi) & =\frac{\mathcal{F}_{0}}{r^{a}}+\frac{\mathcal{F}_{1}}{r^{a_{1}}}+\cdots, \\
\mathcal{L}\left(F^{2}\right) & =\frac{\mathcal{L}_{0}}{r^{b}}+\frac{\mathcal{L}_{1}}{r^{b_{1}}}+\cdots,
\end{aligned}
$$

For example, $a=2 \Delta$ implies that $\mathcal{F}$ behaves as the minimal coupling of the scalar field. If $b=2(\lambda+1)$, the and NED coupling belongs to the class of the Born-Infeld-like Lagrangians that have the same weak-field behavior as the Maxwell electrodynamics (linear in $F^{2}=-2 N \phi^{\prime 2}$ ).

Solving the equations of motion at different orders of $1 / r$ leads to several branches of solutions, a couple of them contain the minimal scalar coupling and Born-Infeld-like theories. A complete set of asymptotic solutions will be discussed in detail in a classification of holographic superconductors [89].

Let us mention some common features of all solutions, mostly independent on the dynamics. The scalar field behaves as $\Psi \sim r^{-(d-\Delta)} \Psi_{\text {source }}$ for large $r$. A dual operator $\mathcal{O}_{\Psi}$ coupled to the scalar in a holographic $\mathrm{CFT}_{d}$ has, therefore, a conformal dimension $\operatorname{dim} \mathcal{O}_{\Psi}=\Delta$ that must be greater than the CFT unitarity bound, $\operatorname{dim} O_{\Psi} \geq \frac{d-2}{2}$ [90]. The equation of motion (25) gives a well-known relation that determines $\Delta$ in terms of the scalar mass,

$\Delta(\Delta-d)=m^{2} \ell_{\mathrm{eff}}^{2}$, 
where it was assumed that there is no conformal anomaly, that is, $\Delta \neq d$, and the electromagnetic interaction does not modify the asymptotic sector, $a>2 \Delta-2$. This equation leads to two branches for the scalar field, $\Delta=\Delta_{+}$and $\Delta=\Delta_{-}=d-\Delta_{+}$. If $d-\Delta<0$, the scalar field is divergent in IR sector and the dual operator with $\operatorname{dim} \mathcal{O}_{\Psi}>d$ is irrelevant deformation of the theory. If the scalar field falls off sufficiently fast $(d-\Delta \geq 0), \mathcal{O}_{\Psi}$ is relevant or a marginal operator satisfying $\operatorname{dim} \mathcal{O}_{\Psi} \leq d$, and it can be turned on without destroying the UV fixed point of the dual $\mathrm{QFT}_{d}$. We are interested in the last case because we want to interpret $\Psi(r)$ as the black hole hair, so we need it to be regular everywhere, including the IR region. These conditions allow for a tachyonic scalar field that still gives unitary CFT, known as the Breitenlohner-Freedman window [91,92]. As regards the scalar observable in $\mathrm{QFT}_{d}$ that will play the role of the order parameter of a superconductor, it can be only a normalizable mode. It is common to take the source to be switched off, $\Psi_{\text {source }}=0$, and then $\Psi_{0}=\left\langle\mathcal{O}_{\Psi}\right\rangle$ is a normalizable operator $[18,51,52]$ and it is of the leading order in the near-boundary expansion, as presented in (87).

The first integral of the NED equation (26) gives an integration constant that is related to the Noether charge,

$4 r^{d-1} \sqrt{N} E \frac{d \mathcal{L}}{d F^{2}}=-\frac{2 \kappa^{2} Q}{V_{d-1}}+\int d r \frac{r^{d-1} \mathcal{F} \phi}{\sqrt{N} f}$.

Non-vanishing $Q$ fixes the fall-off of the electric potential as $\lambda=b-d$. The source $\phi_{0}$ is, then, an algebraic function of the electric charge, $Q=Q\left(\phi_{0}\right)$, given by

$\frac{\mathcal{L}_{0}}{\phi_{0}}=\frac{2 \kappa^{2}(b-d)(b-d+1)}{b V_{d-1}} Q$.

Similarly, the first integral of the gravitational equation $\mathcal{E}_{t}^{t}$ gives rise to an integration constant that depends on the black hole mass obtained from the Noether formula (69),

$$
\begin{gathered}
r^{d-2} N f-\alpha(d-2)(d-3) r^{d-4} N^{2} f^{2}-\frac{r^{d}}{\ell^{2}} \\
=-\frac{2 \kappa^{2} M}{(d-1) V_{d-1}}+\int d r \frac{2 r^{d-1}}{d-1} T_{t}^{t}(r) .
\end{gathered}
$$

The requirement $M \neq 0$ determines the leading order of this equation as $u=d$ and also imposes the requirement that the subleading order of $N(r)$ must be small enough, $s_{1}>d$. The integration constant is then related to the coefficient $f_{0}$ by

$$
f_{0}=-\frac{2 \kappa^{2} \ell_{\mathrm{eff}}^{2}}{(d-1) V_{d-1}\left(1-\frac{2 \alpha}{\ell_{\mathrm{eff}}^{2}}(d-2)(d-3)\right)} M .
$$

It is worthwhile noticing that this is not the unique way to obtain $M \neq 0$ from the asymptotic expansion-other branches can involve the scalar field contribution and even the electric charge. However, it remains to analyze how these new branches modify the UV sector of dual QFT.

We shall not write here the subleading orders of the solutions. Each order of the field equations solves one of the coefficients $X_{n}$ as polynomials in $f_{0}, \phi_{0}$ and $\psi_{0}$, that is, $M, Q$ and $\left\langle\mathcal{O}_{\Psi}\right\rangle$. Furthermore, imposing the boundary conditions on the horizon will involve also the parameters $T$ and $r_{+}$and eliminate the mass as an independent variable. As a result, using the asymptotic solution, the free energy $F\left(T, Q,\left\langle\mathcal{O}_{\Psi}\right\rangle\right)$ can be cast into the form

$$
\tilde{F}\left(r_{+}, \phi_{0}, \Psi_{0}\right)=F\left(T\left(r_{+}, \phi_{0}, \Psi_{0}\right), Q\left(\phi_{0}\right), \Psi_{0}\right) .
$$

An analysis of the extrema of this function for constant $Q$ and $T$ is then a well-posed problem.

\section{Conclusions}

Motivated by an application of AdS/CFT correspondence to $d$-dimensional high- $\mathrm{T}_{c}$ superconductors that do not have a generally accepted theoretical model, we study the Stückelberg scalar field in $(d+1)$-dimensional asymptotically AdS spacetime coupled to gravitational and electromagnetic fields. On the $\operatorname{AdS}_{d+1}$ gravity side, the black hole solution is associated to a thermal, dual $\mathrm{QFT}_{d}$ and the scalar field couples to the order parameter of the superconductor in $\mathrm{QFT}_{d}$.

In order to involve a wider class of holographic superconductors, we include nonlinear effects in the gravity action: the GB term and LL generalization of general relativity that is polynomial in the Riemann curvature and has $[d / 2]$ coupling constants, NED field described by an arbitrary function $\mathcal{L}\left(F^{2}\right)$ and the Stückelberg modification of the scalar field kinetic term through the function $\mathcal{F}(\Psi)$. Of course, because we are ultimately interested in lower-dimensional QFTs, one should also introduce other higher-order curvature terms not of Lovelock type.

On the other hand, study of phase transitions in QFTs can teach us about instabilities of black hole solutions in LL-AdS gravities, as well.

We focus to the maximally symmetric, charged, AdS black holes with flat horizons. Using the Kounterterm regularization of the AdS gravity action that is universal for all LL gravities, we obtain the exact formula for the IR divergence-free Euclidean action and the finite Noether charges. Depending on the choice of the NED boundary term, the Euclidean action is identified with the appropriate thermodynamic potential in canonical or grand canonical ensemble. These thermodynamic potentials, obtained from the gravitational quantum statistical relation, correctly reproduce the Legendre transformation of the internal energy of the superconductor in a dual QFT. 
We also note that the effect of the backreaction cancels all divergences in the scalar field. Furthermore, the Stückelberg scalar does not contribute to the black hole mass. As regards the entropy, it still respects the horizon area law because, in the flat transversal section, there is no LL contribution to the Euclidean action, and the Stückelberg field does not induce extra terms that were noted for other kinds of non-minimal couplings of the scalar field.

Let us emphasize that the novelty of our analytic method is that it provides a holographic free energy formula for the Stückelberg superconductor without using any approximation. The phase transitions now can be analyzed as in the Ginzburg-Landau model, that is, by studying its extrema. This technique can also be applied to 5D Einstein-Hilbert AdS gravity. However, thanks to the regularization method employed, the formula is extended to all LL-AdS gravities in higher dimensions.

A natural direction for future research is to discuss how these results can be used to obtain physical information about a holographic superconductor. More explicitly, the study of local and global minima of the free energy of a holographic superconductor can identify all possible discontinuities of second order associated to phase transitions. This method would enable one to classify all LL AdS superconductors in the space of parameters. By looking only at the asymptotic solutions in the leading and subleading orders, it is possible to obtain information as regards the critical temperature and critical exponent. As concluding remarks, we discuss the general features of the asymptotic solutions.

Acknowledgments The authors thank Rodrigo Olea and Dumitru Astefanesei for useful comments. L.A. would also like to thank Stefan Theisen for hospitality during her stay at Max Planck Institute for Gravitational Physics (Albert Einstein Institute). This work was supported by Chilean FONDECYT grant Nb.1110102. O.M. is grateful to DII-PUCV for their support through the project Nb.123.711/2011. The work of L.A. is financed in part by Chilean grants NAC-doctorado Nb.21090754, BCH-pasantia doctoral Nb.75120006, and the UTFSM projects PIIC/2011-2013.

Open Access This article is distributed under the terms of the Creative Commons Attribution License which permits any use, distribution, and reproduction in any medium, provided the original author(s) and the source are credited.

Funded by $\mathrm{SCOAP}^{3}$ / License Version CC BY 4.0.

\section{Appendix A: Black hole ansatz identities}

The metric of the maximally symmetric, planar black hole in a spacetime with local coordinates $x^{\mu}=\left(t, r, y^{m}\right)$ has the form

$g_{\mu \nu}=\left(\begin{array}{ccc}-f(r) & 0 & 0 \\ 0 & \frac{1}{f(r) N(r)} & 0 \\ 0 & 0 & r^{2} \delta_{m n}\end{array}\right), \quad \sqrt{-g}=\frac{r^{d-1}}{\sqrt{N}}$, where $f(r) \geq 0$ and $N(r)>0$. In this ansatz, the Riemann tensor $R_{\lambda \rho}^{\mu \nu}=R_{\sigma \lambda \rho}^{\mu} g^{\sigma v}$ has non-vanishing components

$R_{t r}^{t r}=-\frac{1}{2}\left(N f^{\prime \prime}+\frac{1}{2} f^{\prime} N^{\prime}\right), \quad R_{t m}^{t n}=-\frac{N f^{\prime}}{2 r} \delta_{m}^{n}$,

$R_{m_{1} m_{2}}^{n_{1} n_{2}}=-\frac{f N}{r^{2}} \delta_{m_{1} m_{2}}^{n_{1} n_{2}}, \quad R_{r m}^{r n}=-\frac{(f N)^{\prime}}{2 r} \delta_{m}^{n}$,

plus the components obtained from the antisymmetry in the pairs of indices. The prime denotes the radial derivative.

The components of the Ricci tensor, $R_{v}^{\mu}=R_{v \lambda}^{\mu \lambda}$, are given by

$$
\begin{aligned}
& R_{t}^{t}=-\frac{1}{2 r}\left[r N f^{\prime \prime}+\frac{1}{2} r f^{\prime} N^{\prime}+(d-1) N f^{\prime}\right], \\
& R_{r}^{r}=-\frac{1}{2 r}\left[r N f^{\prime \prime}+\frac{1}{2} r f^{\prime} N^{\prime}+(d-1)(f N)^{\prime}\right], \\
& R_{m}^{n}=-\frac{1}{r^{2}} \delta_{m}^{n}\left[r N f^{\prime}+\frac{1}{2} r f N^{\prime}+(d-2) f N\right] .
\end{aligned}
$$

The Ricci scalar, $R=R_{\mu}^{\mu}$, then reads

$$
\begin{aligned}
R= & -\frac{1}{r^{2}} N\left[r^{2} f^{\prime \prime}+2(d-1) r f^{\prime}+(d-1)(d-2) f\right] \\
& -\frac{1}{2 r} N^{\prime}\left[r f^{\prime}+2(d-1) f\right]
\end{aligned}
$$

and the GB term is

$$
\begin{aligned}
\frac{1}{4} \delta_{v_{1} \ldots \nu_{4}}^{\mu_{1} \ldots \mu_{4}} R_{\mu_{1} \mu_{2}}^{\nu_{1} \nu_{2}} R_{\mu_{3} \mu_{4}}^{\nu_{3} v_{4}}= & \frac{2(d-1)(d-2)}{r^{4}} \\
& \times\left[N N^{\prime}\left((d-3) r f^{2}+\frac{3}{2} r^{2} f f^{\prime}\right)\right. \\
& +N^{2}\left(r^{2} f f^{\prime \prime}+r^{2} f^{\prime 2}+2(d-3) r f f^{\prime}\right. \\
& \left.\left.+\frac{1}{2}(d-3)(d-4) f^{2}\right)\right]
\end{aligned}
$$

The components of the Einstein tensor with the negative cosmological constant have the form

$$
\begin{aligned}
G_{r}^{r}= & \frac{d-1}{2 r^{2}}\left[r N f^{\prime}+(d-2) N f-d \frac{r^{2}}{\ell^{2}}\right], \\
G_{t}^{t}= & G_{r}^{r}+\frac{d-1}{2 r} N^{\prime} f, \\
G_{m}^{n}= & \frac{1}{2 r^{2}} \delta_{m}^{n}\left[r^{2} N f^{\prime \prime}+2(d-2) r N f^{\prime}+\frac{1}{2} r^{2} N^{\prime} f^{\prime}\right. \\
& \left.+(d-2) r N^{\prime} f+(d-2)(d-3) N f-d(d-1) \frac{r^{2}}{\ell^{2}}\right],
\end{aligned}
$$


and the Lanczos tensor in components reads

$$
\begin{aligned}
H_{r}^{r}= & -\alpha(d-1)(d-2)(d-3) \frac{f}{r^{3}} N^{2}\left(f^{\prime}+\frac{d-4}{2 r} f\right), \\
H_{t}^{t}= & H_{r}^{r}-\alpha(d-1)(d-2)(d-3) \frac{f^{2} N N^{\prime}}{r^{3}}, \\
H_{m}^{n}= & -\alpha(d-2)(d-3) \delta_{m}^{n} \frac{1}{r^{2}}\left[N^{2} f f^{\prime \prime}+N^{2} f^{\prime 2}\right. \\
& +\frac{3}{2} N N^{\prime} f f^{\prime}+\frac{1}{r}(d-4)\left(2 N^{2} f f^{\prime}+N N^{\prime} f^{2}\right) \\
& \left.+(d-4)(d-5) \frac{f^{2} N^{2}}{2 r^{2}}\right] .
\end{aligned}
$$

We also write the following auxiliary expressions:

$$
\begin{aligned}
G_{m}^{m} & =\frac{\left(r^{d-1} G_{r}^{r}\right)^{\prime}}{r^{d-2}}-\frac{d-1}{4} N^{\prime} f^{\prime}, \\
H_{m}^{m} & =\frac{\left(r^{d-1} H_{r}^{r}\right)^{\prime}}{r^{d-2}}+\alpha(d-1)(d-2)(d-3) \frac{N N^{\prime} f f^{\prime}}{2 r^{2}} .
\end{aligned}
$$

\section{Appendix B: Ordinary hypergeometric function}

The ordinary hypergeometric function in the integral representation is given by

$$
\begin{aligned}
& { }_{2} F_{1}(a, b ; c ; z) \\
& =\frac{\Gamma(c)}{\Gamma(b) \Gamma(c-b)} \int_{0}^{1} d t \frac{t^{b-1}(1-t)^{c-b-1}}{(1-z t)^{a}}, \quad \Re(c)>\Re(b)>0 .
\end{aligned}
$$

It can be expanded in the hypergeometric series whose coefficients are given by the Pochhammer symbol, $(a)_{p}$,

$$
\begin{aligned}
{ }_{2} F_{1}(a, b ; c ; z) & =1+\frac{a b}{c} z+\frac{a(a+1) b(b+1)}{2 c(c+1)} z^{2}+\cdots \\
& =\sum_{p=0}^{\infty} \frac{(a)_{p}(b)_{p}}{(c)_{p}} \frac{z^{p}}{p !},
\end{aligned}
$$

which converges when $c$ is not a negative integer for all $|z|<$ 1 , and on the unit circle $|z|=1$ if $\Re(c-a-b)>0$.

In this text, we need the following integrals represented in terms of the hypergeometric functions:

$$
\begin{aligned}
\int_{0}^{1} d u\left(-\beta+u^{2} w\right)^{n-1} & =(-\beta)^{n-1}{ }_{2} F_{1}\left(1-n, \frac{1}{2} ; \frac{3}{2} ; \frac{w}{\beta}\right) \\
\int_{0}^{1} d s\left(1-s^{2}\right)^{n-1} & ={ }_{2} F_{1}\left(1-n, \frac{1}{2} ; \frac{3}{2} ; 1\right) \\
& =\frac{2^{2 n-2}(n-1) !^{2}}{(2 n-1) !},
\end{aligned}
$$

as well as the integrals used in Sects. 5 and 6,

$$
\begin{aligned}
\mathcal{I}_{n}(\beta, w)= & \int_{0}^{1} d u \int_{0}^{u} d s\left[-u^{2} \beta+(2 n-1) s^{2} w\right] \\
& \times\left(-u^{2} \beta+s^{2} w\right)^{n-2}=\frac{(w-\beta)^{n-1}}{2 n} \\
\mathcal{J}_{n}(\beta, w)= & \int_{0}^{1} d u \int_{0}^{u} d s\left[-(2 n-1) u^{2} \beta+s^{2} w\right] \\
= & \left(-u^{2} \beta+s^{2} w\right)^{n-2}(-\beta)^{n-1} F_{1} \\
& \times\left(1-n, \frac{1}{2} ; \frac{3}{2} ; \frac{w}{\beta}\right)-\frac{(w-\beta)^{n-1}}{2 n} .
\end{aligned}
$$

\section{References}

1. J.M. Maldacena, The large $N$ limit of superconformal field theories. Adv. Theor. Math. Phys. 2, 231 (1998)

2. J.M. Maldacena, The large $N$ limit of superconformal field theories. Int. J. Theor. Phys. 38, 1113 (1999). hep-th/9711200

3. S.S. Gubser, I.R. Klebanov, A.M. Polyakov, A semiclassical limit of the gauge string correspondence. Nucl. Phys. B 636, 99 (2002).hep-th/0204051

4. E. Witten, Anti-de Sitter space and holography. Adv. Theor. Math. Phys. 2, 253 (1998). hep-th/9802150

5. S.A. Hartnoll, C.P. Herzog, G.T. Horowitz, Building a holographic superconductor. Phys. Rev. Lett. 101, 031601 (2008). arXiv:0803.3295 [hep-th]

6. S.A. Hartnoll, C.P. Herzog, G.T. Horowitz, Holographic superconductors. JHEP 0812, 015 (2008). arXiv:0810.1563 [hep-th]

7. E.C.G. Stückelberg, Interaction forces in electrodynamics and in the field theory of nuclear forces. Helv. Phys. Acta 11, 299 (1938). (in German)

8. S. Chen, L. Wang, C. Ding, J. Jing, Holographic superconductors in the AdS black hole spacetime with a global monopole. Nucl. Phys. B 836, 222 (2010). arXiv:0912.2397 [gr-qc]

9. S. Franco, A. Garcia-Garcia, D. Rodriguez-Gomez, A general class of holographic superconductors. JHEP 1004, 092 (2010). arXiv:0906.1214 [hep-th]

10. S. Franco, A.M. Garcia-Garcia, D. Rodriguez-Gomez, A holographic approach to phase transitions. Phys. Rev. D 81, 041901 (2010). arXiv:0911.1354 [hep-th]

11. D.Z. Ma, Y. Cao, J.P. Wu, The St ückelberg holographic superconductors with Weyl corrections. Phys. Lett. B 704, 604 (2011). arXiv: 1201.2486 [hep-th]

12. S.S. Gubser, Breaking an Abelian gauge symmetry near a black hole horizon. Phys. Rev. D 78, 065034 (2008). arXiv:0801.2977 [hep-th]

13. S.S. Gubser, Colorful horizons with charge in anti-de Sitter space. Phys. Rev. Lett. 101, 191601 (2008). arXiv:0803.3483 [hep-th]

14. S. Gangopadhyaya, D. Roychowdhury, Analytic study of properties of holographic superconductors in Born-Infeld electrodynamics. JHEP 1205, 002 (2012). arXiv: 1201.6520 [hep-th]

15. D. Roychowdhury, Effect of external magnetic field on holographic superconductors in presence of nonlinear corrections. Phys. Rev. D 86, 106009 (2012). arXiv:1211.0904 [hep-th]

16. D. Momeni, N. Majd, R. Myrzakulov, p-Wave holographic superconductors with Weyl corrections. Europhys. Lett. 97, 61001 (2012). arXiv:1204.1246 [hep-th] 
17. S.S. Gubser, S.S. Pufu, The gravity dual of a p-wave superconductor. JHEP 0811, 033 (2008). arXiv:0805.2960 [hep-th]

18. C.P. Herzog, An analytic holographic superconductor. Phys. Rev. D 81, 126009 (2010). arXiv: 1003.3278 [hep-th]

19. X.H. Ge, Analytical calculation on critical magnetic field in holographic superconductors with backreaction. Prog. Theor. Phys. 128, 1211 (2012). arXiv:1105.4333 [hep-th]

20. I.R. Klebanov, E. Witten, AdS/CFT correspondence and symmetry breaking. Nucl. Phys. B 556, 89 (1999). hep-th/9905104

21. C. Lanczos, A remarkable property of the Riemann-Christoffel tensor in four dimensions. Ann. Math. 39, 842 (1938)

22. D. Lovelock, The Einstein tensor and its generalizations. J. Math. Phys. 12, 498 (1971)

23. D. Lovelock, The four-dimensionality of space and the Einstein tensor. J. Math. Phys. 13, 874 (1972)

24. J. Jing, L. Wang, Q. Pan, S. Chen, Holographic superconductors in Gauss-Bonnet gravity with Born-Infeld electrodynamics. Phys. Rev. D 83, 066010 (2011). arXiv:1012.0644 [gr-qc]

25. J. Jing, Q. Pan, S. Chen, Holographic superconductor/insulator transition with logarithmic electromagnetic field in Gauss-Bonnet gravity. Phys. Lett. B 716, 385 (2012). arXiv:1209.0893 [hep-th]

26. Q. Pan, J. Jing, B. Wang, Analytical investigation of the phase transition between holographic insulator and superconductor in GaussBonnet gravity. JHEP 1111, 088 (2011). arXiv:1105.6153 [gr-qc]

27. S. Kanno, A note on Gauss-Bonnet holographic superconductors. Class. Quantum Grav. 28, 127001 (2011). arXiv:1103.5022 [hepth]

28. M.R. Setare, D. Momeni, Gauss-Bonnet holographic superconductors with magnetic field. Europhys. Lett. 96, 60006 (2011). arXiv:1106.1025 [physics.gen-ph]

29. R. Gregory, Holographic superconductivity with Gauss-Bonnet gravity. J. Phys. Conf. Ser. 283, 012016 (2011). arXiv:1012.1558 [hep-th]

30. Y. Liu, Y. Peng, B. Wang, Gauss-Bonnet holographic superconductors in Born-Infeld electrodynamics with backreactions. arXiv:1202.3586 [hep-th]

31. S. Gangopadhyay, Analytic study of properties of holographic superconductors away from the probe limit. arXiv:1302.1288 [hepth]

32. L. Barclay, R. Gregory, S. Kanno, P. Sutcliffe, GaussBonnet holographic superconductors. JHEP 1012, 029 (2010). arXiv:1009.1991 [hep-th]

33. G. Siopsis, J. Therrien, Analytic calculation of properties of holographic superconductors. JHEP 1005, 013 (2010). arXiv:1003.4275 [hep-th]

34. L. Barclay, The rich structure of Gauss-Bonnet holographic superconductors. JHEP 1110, 044 (2011). arXiv:1012.3074 [hep-th]

35. Q. Pan, B. Wang, General holographic superconductor models with Gauss-Bonnet corrections. Phys. Lett. B 693, 159 (2010). arXiv: 1005.4743 [hep-th]

36. R.G. Cai, Z.Y. Nie, H.Q. Zhang, Holographic p-wave superconductors from Gauss-Bonnet gravity. Phys. Rev. D 82, 066007 (2010). arXiv:1007.3321 [hep-th]

37. R.G. Cai, Z.Y. Nie, H.Q. Zhang, Holographic phase transitions of p-wave superconductors in Gauss-Bonnet gravity with backreaction. Phys. Rev. D 83, 066013 (2011). arXiv:1012.5559 [hepth]

38. H.F. Li, R.G. Cai, H.Q. Zhang, Analytical studies on holographic superconductors in Gauss-Bonnet gravity. JHEP 1104, 028 (2011). arXiv:1103.2833 [hep-th]

39. Y. Liu, Q. Pan, B. Wang, R.G. Cai, Dynamical perturbations and critical phenomena in Gauss-Bonnet-AdS black holes. Phys. Lett. B 693, 343 (2010). arXiv:1007.2536 [hep-th]

40. S. Gangopadhyaya, D. Roychowdhury, Analytic study of GaussBonnet holographic superconductors in Born-Infeld electrodynamics. JHEP 1205, 156 (2012). arXiv:1204.0673 [hep-th]
41. A. Buchel, J.T. Liu, Universality of the shear viscosity in supergravity. Phys. Rev. Lett. 93, 090602 (2004). hep-th/0311175

42. X.-H. Ge, S.-J. Sin, S.-F. Wu, G.-H. Yang, Shear viscosity and instability from third order Lovelock gravity. Phys. Rev. D 80, 104019 (2009). arXiv:0905.2675 [hep-th]

43. A. Salvio, Holographic superfluids and superconductors in dilatongravity. JHEP 1209, 134 (2012). arXiv:1207.3800 [hep-th]

44. J. Jing, Q. Pan, S. Chen, Holographic superconductors with PowerMaxwell field. JHEP 1111, 045 (2011). arXiv:1106.5181 [hep-th]

45. O. Domenech, M. Montull, A. Pomarol, A. Salvio, P.J. Silva, Emergent gauge fields in holographic superconductors. JHEP 1008, 033 (2010). arXiv:1005.1776 [hep-th]

46. X.-H. Ge, B. Wang, S.-F. Wu, G.-H. Yang, Analytical study on holographic superconductors in external magnetic field. JHEP 1008, 108 (2010). arXiv:1002.4901 [hep-th]

47. Q. Pan, J. Jing, B. Wang, S. Chen, Analytical study on holographic superconductors with backreactions. JHEP 1206, 087 (2012). arXiv:1205.3543 [hep-th]

48. D. Roychowdhury, AdS/CFT superconductors with Power Maxwell electrodynamic: reminiscent of the Meissner effect. Phys. Lett. B 718, 1089 (2013). arXiv:1211.1612 [hep-th]

49. N. Bai, Y. Gao, B.G. Qi, X.B. Xu, Holographic insulator/superconductor phase transition in Born-Infeld electrodynamics. arXiv:1212.2721 [hep-th]

50. D. Momeni, R. Myrzakulov, M. Raza, Holographic superconductors with Weyl Corrections via gauge/gravity duality. arXiv: 1307.8348 [hep-th]

51. S.A. Hartnoll, Lectures on holographic methods for condensed matter physics. Class. Quantum Grav. 26, 224002 (2009). arXiv:0903.3246 [hep-th]

52. C.P. Herzog, Lectures on holographic superfluidity and superconductivity. J. Phys. A 42, 343001 (2009). arXiv:0904.1975 [hep-th]

53. J. de Boer, M. Kulaxizi, A. Parnachev, Holographic Lovelock gravities and black holes. JHEP 1006, 008 (2010). arXiv:0912.1877 [hep-th]

54. X.O. Camanho, J.D. Edelstein, Causality in AdS/CFT and Lovelock theory. JHEP 1006, 099 (2010). arXiv:0912.1944 [hep-th]

55. X.O. Camanho, J.D. Edelstein, M.F. Paulos, Lovelock theories, holography and the fate of the viscosity bound. JHEP 1105, 127 (2011). arXiv:1010.1682 [hep-th]

56. R.-G. Cai, Gauss-Bonnet black holes in AdS spaces. Phys. Rev. D 65, 084014 (2002). hep-th/0109133

57. C. Garraffo, G. Giribet, The Lovelock black holes. Mod. Phys. Lett. A 23, 1801 (2008). arXiv:0805.3575 [gr-qc]

58. X.O. Camanho, J.D. Edelstein, A Lovelock black hole bestiary. Class. Quantum Grav. 30, 035009 (2013). arXiv:1103.3669 [hepth]

59. N. Dadhich, J.M. Pons, K. Prabhu, On the static Lovelock black holes. Gen. Rel. Grav. 45, 1131 (2013). arXiv:1201.4994 [gr-qc]

60. M. Aiello, R. Ferraro, G. Giribet, Exact solutions of LovelockBorn-Infeld black holes. Phys. Rev. D 70, 104014 (2004). gr-qc/0408078

61. R.C. Myers, J.Z. Simon, Black hole thermodynamics in Lovelock gravity. Phys. Rev. D 38, 2434 (1988)

62. R.-G. Cai, A note on thermodynamics of black holes in Lovelock gravity. Phys. Lett. B 582, 237 (2004). hep-th/0311240

63. T. Padmanabhan, D. Kothawala, Lanczos-Lovelock models of gravity. arXiv:1302.2151 [gr-qc]

64. J.D. Edelstein, Lovelock theory, black holes and holography. arXiv:1303.6213 [gr-qc]

65. D.G. Boulware, S. Deser, String generated gravity models. Phys. Rev. Lett. 55, 2656 (1985)

66. S. Deser, B. Tekin, Gravitational energy in quadratic curvature gravities. Phys. Rev. Lett. 89, 101101 (2002). hep-th/0205318

67. C. Charmousis, A. Padilla, The instability of vacua in GaussBonnet gravity. JHEP 0812, 038 (2008). arXiv:0807.2864 [hep-th] 
68. A.H. Chamseddine, Topological gravity and supergravity in various dimensions. Nucl. Phys. B 346, 213 (1990)

69. O. Miskovic, R. Troncoso, J. Zanelli, Canonical sectors of fivedimensional Chern-Simons theories. Phys. Lett. B 615, 277 (2005). hep-th/0504055

70. O. Miskovic, R. Olea, Conserved charges for black holes in Einstein-Gauss-Bonnet gravity coupled to nonlinear electrodynamics in AdS space. Phys. Rev. D 83, 024011 (2011). arXiv: 1009.5763 [hep-th]

71. M. Born, L. Infeld, Foundations of the new field theory. Proc. R. Soc. Lond. A 144, 425 (1934)

72. O. Miskovic, R. Olea, Thermodynamics of Einstein-Born-Infeld black holes with negative cosmological constant. Phys. Rev. D 77, 124048 (2008). arXiv:0802.2081 [hep-th]

73. R. Gregory, S. Kanno, J. Soda, Holographic superconductors with higher curvature corrections. JHEP 0910, 010 (2009). arXiv:0907.3203 [hep-th]

74. S. de Haro, S.N. Solodukhin, K. Skenderis, Holographic reconstruction of space-time and renormalization in the AdS/CFT correspondence. Commun. Math. Phys. 217, 595 (2001). hep-th/0002230

75. C. Fefferman, R. Graham, in Conformal Invariants, The Mathematical Heritage of Elie Cartan (Lyon 1984), Asté risque, 1985, Numero Hors Serie, vol 95

76. A. Schwimmer, S. Theisen, Diffeomorphisms, anomalies and the Fefferman-Graham ambiguity. JHEP 0008, 032 (2000). hep-th/0008082

77. M. Henningson, K. Skenderis, The holographic Weyl anomaly. JHEP 9807, 023 (1998). hep-th/9806087

78. G. Kofinas, R. Olea, Universal regularization prescription for Lovelock AdS gravity. JHEP 0711, 069 (2007). arXiv:0708.0782 [hepth]

79. G. Kofinas, R. Olea, Universal Kounterterms in Lovelock AdS gravity. Fortsch. Phys. 56, 957 (2008). arXiv:0806.1197 [hep-th]

80. O. Miskovic, R. Olea, Counterterms in dimensionally continued AdS gravity. JHEP 0710, 028 (2007). arXiv:0706.4460 [hep-th]
81. G. Kofinas, R. Olea, Vacuum energy in Einstein-Gauss-Bonnet AdS gravity. Phys. Rev. D 74, 084035 (2006). hep-th/0606253

82. R. Olea, Regularization of odd-dimensional AdS gravity: Kounterterms. JHEP 0704, 073 (2007). hep-th/0610230

83. M.M. Caldarelli, G. Cognola, D. Klemm, Thermodynamics of Kerr-Newman-AdS black holes and conformal field theories. Class. Quantum Grav. 17, 399 (2000). arXiv:hep-th/9908022

84. G.W. Gibbons, M.J. Perry, C.N. Pope, The first law of thermodynamics for Kerr-anti-de Sitter black holes. Class. Quantum Grav. 22, 1503 (2005). hep-th/0408217

85. A. Ashtekar, A. Corichi, D. Sudarsky, Nonminimally coupled scalar fields and isolated horizons. Class. Quantum Grav. 20, 3413 (2003). gr-qc/0305044

86. O. Miskovic, R. Olea, Quantum statistical relation for black holes in nonlinear electrodynamics coupled to Einstein-Gauss-Bonnet AdS gravity. Phys. Rev. D 83, 064017 (2011). arXiv:1012.4867 [hep-th]

87. O. Miskovic, R. Olea, Thermodynamics of black holes in EinsteinGauss-Bonnet AdS gravity coupled to nonlinear electrodynamics. J. Phys. Conf. Ser. 343, 012077 (2012)

88. Y.S. Myung, C. Park, Holographic superconductor in the exact hairy black hole. Phys. Lett. B 704, 242 (2011). arXiv:1007.0816 [hep-th]

89. L. Aranguiz, O. Miskovic, Classification of holographic high$T_{c}$ superconductors in Lovelock AdS gravities coupled to NED. Manuscript in preparation

90. S. Minwalla, Restrictions imposed by superconformal invariance on quantum field theories. Adv. Theor. Math. Phys. 2, 781 (1998). hep-th/9712074

91. P. Breitenlohner, D.Z. Freedman, Positive energy in anti-De Sitter backgrounds and gauged extended supergravity. Phys. Lett. B 115, 197 (1982)

92. P. Breitenlohner, D.Z. Freedman, Stability in gauged extended supergravity. Ann. Phys. 144, 249 (1982) 